\title{
Solutions and positive solutions for superlinear Robin problems
}

\author{
Cite as: J. Math. Phys. 60, 101506 (2019); doi: 10.1063/1.5118760 \\ Submitted: 4 July 2019 - Accepted: 16 September 2019 • \\ Published Online: 14 October 2019
}

\author{
N. S. Papageorgiou, ${ }^{1, a)}$ C. Vetro, ${ }^{2, b)}$ (i) and F. Vetro ${ }^{3, c)}$ (i)
}

\begin{abstract}
AFFILIATIONS
${ }^{1}$ Department of Mathematics, National Technical University, Zografou Campus, 15780 Athens, Greece

${ }^{2}$ Department of Mathematics and Computer Science, University of Palermo, Via Archirafi 34, 90123 Palermo, Italy

${ }^{3}$ Nonlinear Analysis Research Group, Ton Duc Thang University, Ho Chi Minh City, Vietnam

and Faculty of Mathematics and Statistics, Ton Duc Thang University, Ho Chi Minh City, Vietnam
\end{abstract}

a) Electronic mail: npapg@math.ntua.gr

${ }^{b}$ Electronic mail: calogero.vetro@unipa.it

c) Author to whom correspondence should be addressed: francescavetro@tdtu.edu.vn

\begin{abstract}
We consider nonlinear, nonhomogeneous Robin problems with a $(p-1)$-superlinear reaction term, which need not satisfy the AmbrosettiRabinowitz condition. We look for positive solutions and prove existence and multiplicity theorems. For the particular case of the $p$-Laplacian, we prove existence results under a different geometry near the origin.
\end{abstract}

Published under license by AIP Publishing. https://doi.org/10.1063/1.5118760

\section{INTRODUCTION}

Let $\Omega \subseteq \mathbb{R}^{N}$ be a bounded domain with a $C^{2}$-boundary $\partial \Omega$. In this work, we study the following nonlinear, nonhomogeneous Robin problem:

$$
\left\{\begin{array}{cl}
-\operatorname{div} a(\nabla u(z))+\xi(z)|u(z)|^{p-2} u(z)=f(z, u(z)) & \text { in } \Omega \\
\frac{\partial u}{\partial n_{a}}+\beta(z)|u|^{p-2} u=0 & \text { on } \partial \Omega .
\end{array}\right.
$$

In this problem, $a: \mathbb{R}^{N} \rightarrow \mathbb{R}^{N}$ is a monotone continuous map, which satisfies certain other regularity and growth conditions, listed in hypotheses $H(a)_{1}$. These conditions are general enough to incorporate in our framework many nonlinear differential operators of interest, such as the $p$-Laplacian $(1<p<\infty)$ and the $(p, q)$-Laplacian $(1<q<p<\infty)$. The potential function $\xi \in L^{\infty}(\Omega)$ is, in general, indefinite [that is, $\xi(\cdot)$ is sign changing]. The reaction term $f(z, x)$ is a Carathéodory function [that is, for all $x \in \mathbb{R}, z \rightarrow f(z, x)$ is measurable and for a.a. (almost all) $z \in \Omega, x \rightarrow f(z, x)$ is continuous]. We assume that $f(z, \cdot)$ is $(p-1)$-superlinear but without satisfying the usual conditions, in such cases the Ambrosetti-Rabinowitz condition (the AR-condition for short). Instead we employ a less restrictive condition, which fits in our setting $(p-1)$-superlinear functions with slower growth near $\pm \infty$, which fail to satisfy the AR-condition. In the boundary condition, $\frac{\partial u}{\partial n_{a}}$ denotes the generalized directional derivative (conormal derivative) of $u$ defined by extension to $W^{1, p}(\Omega)$ of the map $C^{1}(\bar{\Omega}) \ni u \rightarrow \frac{\partial u}{\partial n_{a}}=(a(\nabla u), n)_{\mathbb{R}^{N}}$, with $n(\cdot)$ being the outward unit normal on $\partial \Omega$. This directional derivative is dictated by the nonlinear Green's identity (see, for example, Ref. 12, p. 210). The boundary coefficient $\beta(\cdot)$ is a non-negative Hölder continuous on $\partial \Omega$ function. If $\beta \equiv 0$, then we recover the Neumann problem. We are looking for positive solutions. In Sec. III, we prove two such results under different geometries near the origin. First, $u=0$ is a local minimizer of the energy (Euler) functional and we prove an existence theorem. Second, instead we assume that $f(z, \cdot)$ admits a $z$-dependent zero on the positive semiaxis and we prove the existence of an ordered pair of positive solutions. We also show the existence of a smallest positive solution. In Sec. IV, we deal with the special case of the $p$-Laplacian differential operator $\left[\right.$ that is, $a(y)=|y|^{p-2} y$ for all $y \in \mathbb{R}^{N}$ with $1<p<\infty]$ and a reaction term near zero stays above the principal eigenvalue of the $p$-Laplacian with the Robin boundary condition. 
So, now $u=0$ is no longer a local minimizer of the energy functional leading to a different geometry for the problem and consequently to a different approach. As a particular case of the existence theorem in Sec. IV, we consider a parametric Robin problem with general potential and prove the existence of a positive smooth solution for every value of the parameter $\lambda>0$, extending this way an earlier such result for semilinear Dirichlet problems by Miyagaki-Souto. ${ }^{26}$ Our existence and multiplicity results in this paper extend in different ways the works of Gao-Tang, ${ }^{11}$ Iturriaga-Souto-Ubilla, ${ }^{20}$ Iturriaga-Lorca-Ubilla, ${ }^{21} \mathrm{Liu},{ }^{23} \mathrm{Li}-Y$ ang, ${ }^{24}$ Miyagaki-Souto, ${ }^{26}$ and Sun. ${ }^{37}$ We also mention the related recent work of Papageorgiou-Smyrlis ${ }^{35}$ on positive solutions of nonlinear logistic equations.

Our method of proof uses variational tools based on the critical point theory together with suitable truncation and perturbation techniques, comparison principles, and Morse theory (critical groups).

\section{MATHEMATICAL BACKGROUND}

Let $X$ be a Banach space. By $X^{*}$, we denote the topological dual of $X$, and by $\langle\cdot, \cdot\rangle$, we denote the duality brackets for the pair $\left(X^{*}, X\right)$. Suppose that $\varphi \in C^{1}(X, \mathbb{R})$. We say that $\varphi$ satisfies the "Cerami condition" (the "C $C$-condition" for short), provided the following property holds: "Every sequence $\left\{u_{n}\right\}_{n \in \mathbb{N}} \subseteq X$ such that $\left\{\varphi\left(u_{n}\right)\right\}_{n \in \mathbb{N}} \subseteq \mathbb{R}$ is bounded and $\left(1+\left\|u_{n}\right\|\right) \varphi^{\prime}\left(u_{n}\right) \rightarrow 0$ in $X^{*}$ as $n \rightarrow+\infty$ admits a strongly convergent subsequence." Using this compactness-type condition on the functional $\varphi$, one can prove a deformation theorem from which follows the minimax theory of the critical values of $\varphi$. Prominent in that theory is the so-called "mountain pass theorem" which we recall here as follows:

Theorem 1. If $\varphi \in C^{1}(X, \mathbb{R})$ satisfies the $C$-condition, there exist $u_{0}, u_{1} \in X$ and $\rho>0$ such that $\left\|u_{1}-u_{0}\right\|>\rho$,

$$
\max \left\{\varphi\left(u_{0}\right), \varphi\left(u_{1}\right)\right\}<\inf \left\{\varphi(u):\left\|u-u_{0}\right\|=\rho\right\}=m_{\rho}
$$

and $c=\inf _{\gamma \in \Gamma} \max _{0 \leq t \leq 1} \varphi(\gamma(t))$ with $\Gamma=\left\{\gamma \in C([0,1], X): \gamma(0)=u_{0}, \gamma(1)=u_{1}\right\}$, then $c \geq m_{\rho}$ and $c$ is a critical value of $\varphi[$ that is, there exists $\widehat{u} \in X$ such that $\varphi^{\prime}(\widehat{u})=0$ and $\left.\varphi(\widehat{u})=c\right]$.

The study of problem (1) will be based on the following spaces:

- $\quad$ The Sobolev space $W^{1, p}(\Omega)$.

- The Banach space $C^{1}(\bar{\Omega})$.

- The boundary Lebesgue spaces $L^{q}(\partial \Omega), 1 \leq q \leq \infty$.

By $\|\cdot\|$, we denote the norm of the Sobolev space $W^{1, p}(\Omega)$ defined by

$$
\|u\|=\left[\|u\|_{p}^{p}+\|\nabla u\|_{p}^{p}\right]^{1 / p} \text { for all } u \in W^{1, p}(\Omega)
$$

The Banach space $C^{1}(\bar{\Omega})$ is an ordered Banach space with the positive (order) cone given by $C_{+}=\left\{u \in C^{1}(\bar{\Omega}): u(z) \geq 0\right.$ for all $\left.z \in \bar{\Omega}\right\}$. This cone has a nonempty interior which is given by the set $D_{+}=\left\{u \in C_{+}: u(z)>0\right.$ for all $\left.z \in \bar{\Omega}\right\}$. On $\partial \Omega$, we consider the $(N-1)$-dimensional Hausdorff (surface) measure $\sigma(\cdot)$. Using this measure on $\partial \Omega$, we can define in the usual way the boundary Lebesgue spaces $L^{q}(\partial \Omega)$ $(1 \leq q \leq \infty)$. From the theory of Sobolev spaces, we know that there exists a unique continuous linear map $\gamma_{0}: W^{1, p}(\Omega) \rightarrow L^{q}(\partial \Omega)$, known as the "trace map," such that $\gamma_{0}(u)=\left.u\right|_{\partial \Omega}$ for all $u \in W^{1, p}(\Omega) \cap C(\bar{\Omega})$. Therefore, the trace map gives meaning to the "boundary values" on $\partial \Omega$ of any Sobolev function. We know that $\gamma_{0}$ is compact in $L^{q}(\partial \Omega)$ for $q \in\left[1, \frac{(N-1) p}{N-p}\right)$ if $N>p$ and into $L^{q}(\partial \Omega)$ for all $q \geq 1$ if $N \leq p$. Moreover, we have $\operatorname{ker} \gamma_{0}=W_{0}^{1, p}(\Omega)$ and $\operatorname{im} \gamma_{0}=W^{\frac{1}{p^{\prime}}, p}(\partial \Omega)$ with $\frac{1}{p}+\frac{1}{p^{\prime}}=1$. From now on, for the sake of notational simplicity, we drop the use of the map $\gamma_{0}$. The restriction of any Sobolev function on $\partial \Omega$ is understood in the sense of traces. Let $\vartheta \in C^{1}(0, \infty)$ with $\vartheta(t)>0$ for all $t>0$ and assume that

$$
\left\{\begin{array}{l}
0<\widehat{c} \leq \frac{\vartheta^{\prime}(t) t}{\vartheta(t)} \leq c_{0} \text { for all } t>0, \\
c_{1} t^{p-1} \leq \vartheta(t) \leq c_{2}\left(t^{s-1}+t^{p-1}\right) \text { for all } t>0 \text {, some } c_{1}, c_{2}>0 \quad 1 \leq s<p .
\end{array}\right.
$$

We introduce the following conditions on the map $a(\cdot)$ [see (1)]:

$H(a): a(y)=a_{0}(|y|) y$ for all $y \in \mathbb{R}^{N}$ with $a_{0}(t)>0$ for all $t>0$ and 
(i) $\quad a_{0} \in C^{1}(0, \infty), t \rightarrow a_{0}(t) t$ is strictly increasing, $a_{0}(t) t \rightarrow 0^{+}$as $t \rightarrow 0^{+}$and $\lim _{t \rightarrow 0^{+}} \frac{a_{0}^{\prime}(t) t}{a_{0}(t)}>-1$;

(ii) there exists $c_{3}>0$ such that $|\nabla a(y)| \leq c_{3} \frac{9(|y|)}{|y|}$ for all $y \in \mathbb{R}^{N} \backslash\{0\}$;

(iii) $\quad(\nabla a(y) \xi, \xi)_{\mathbb{R}^{N}} \geq \frac{9(|y|)}{|y|}|\xi|^{2}$ for all $y \in \mathbb{R}^{N} \backslash\{0\}$, all $\xi \in \mathbb{R}^{N}$.

Remark 1. These conditions on a $(\cdot)$ are dictated by the nonlinear regularity theory of Lieberman ${ }^{22}$ and the nonlinear maximum principle of Pucci-Serrin ${ }^{36}$ (pp. 111 and 120).

We set $G_{0}(t)=\int_{0}^{t} a_{0}(s) s d s$ and define $G(y)=G_{0}(|y|)$ for all $y \in \mathbb{R}^{N}$. Evidently, $G(\cdot)$ is convex and $G(0)=0$. Also, we have

$$
\nabla G(y)=G_{0}^{\prime}(|y|) \frac{y}{|y|}=a_{0}(|y|) y=a(y) \quad \text { for all } y \in \mathbb{R}^{N} \backslash\{0\}
$$

$\nabla G(0)=0$. Therefore, $G(\cdot)$ is the primitive of $a(\cdot)$. Then, the convexity of $G(\cdot)$, and since $G(0)=0$, implies that

$$
G(y) \leq(a(y), y)_{\mathbb{R}^{N}} \quad \text { for all } y \in \mathbb{R}^{N} .
$$

Lemma 1 summarizes the basic properties of the map $a(\cdot)$ and is an easy consequence of hypotheses $H(a)$.

Lemma 1. If hypotheses $H(a)$ hold, then

(a) $\quad y \rightarrow a(y)$ is continuous, strictly monotone, hence maximal monotone too;

(b) $|a(y)| \leq c_{4}\left(1+|y|^{p-1}\right)$ for all $y \in \mathbb{R}^{N}$, some $c_{4}>0$;

(c) $(a(y), y)_{\mathbb{R}^{N}} \geq \frac{c_{1}}{p-1}|y|^{p}$ for all $y \in \mathbb{R}^{N}$.

This lemma together with (3) lead to the following growth estimates for the primitive $G(\cdot)$ :

Corollary 1. If hypotheses $H(a)$ hold, then $\frac{c_{1}}{p(p-1)}|y|^{p} \leq G(y) \leq c_{5}\left(1+|y|^{p}\right)$ for all $y \in \mathbb{R}^{N}$, some $c_{5}>0$.

In addition, we impose the following conditions on the potential function $\xi(\cdot)$ and the boundary coefficient $\beta(\cdot)$ :

$\mathrm{H}(\xi): \xi \in L^{\infty}(\Omega)$.

$\mathrm{H}(\beta): \beta \in C^{0, \alpha}(\partial \Omega)$ for some $\alpha \in(0,1)$ with $\beta(z) \geq 0$ for all $z \in \partial \Omega$.

Remark 2. When $\beta \equiv 0$, we have the usual Neumann problem.

Consider a Carathéodory function $f_{0}: \Omega \times \mathbb{R} \rightarrow \mathbb{R}$ which satisfies

$$
\left|f_{0}(z, x)\right| \leq a_{0}(z)\left(1+|x|^{p^{*}-1}\right) \quad \text { for a.a. } z \in \Omega \text {, all } x \in \mathbb{R}
$$

with $a_{0} \in L^{\infty}(\Omega)_{+}, p^{*}=\left\{\begin{array}{l}\frac{N p}{N-p} \text { if } N>p \\ +\infty \text { if } N \leq p\end{array}\right.$ (the critical Sobolev exponent).

We set $F_{0}(z, x)=\int_{0}^{x} f_{0}(z, s) d s$ and also define the $C^{1}$-functional $\mu: W^{1, p}(\Omega) \rightarrow \mathbb{R}$ by

$$
\mu(u)=\int_{\Omega} p G(\nabla u) d z+\int_{\Omega} \xi(z)|u|^{p} d z+\int_{\partial \Omega} \beta(z)|u|^{p} d \sigma
$$

for all $u \in W^{1, p}(\Omega)$. Then, we consider the $C^{1}$-functional $\varphi_{0}: W^{1, p}(\Omega) \rightarrow \mathbb{R}$ defined by

$$
\varphi_{0}(u)=\frac{1}{p} \mu(u)-\int_{\Omega} F(z, u) d z \quad \text { for all } u \in W^{1, p}(\Omega) .
$$

We set $V=C^{1}(\bar{\Omega})$ and $X=W^{1, p}(\Omega)=\overline{C^{1}(\Omega)}\|\cdot\|$. The following result can be found in Ref. 30 (subcritical case) and Ref. 32 (critical case). 
Proposition 1. If $u_{0} \in W^{1, p}(\Omega)$ is a local $V$-minimizer of $\varphi_{0}$, that is, there exists $\rho_{0}>0$ such that $\varphi_{0}\left(u_{0}\right) \leq \varphi_{0}\left(u_{0}+h\right)$ for all $h \in V$, $\|h\|_{V} \leq \rho_{0}$, then $u_{0} \in C^{1, \alpha}(\bar{\Omega})$ for some $\alpha \in(0,1)$ and $u_{0}$ is a local $X$-minimizer of $\varphi_{0}$, that is, there exists $\rho_{1}>0$ such that $\varphi_{0}\left(u_{0}\right) \leq \varphi_{0}\left(u_{0}+h\right)$ for all $h \in X$, $\|h\| \leq \rho_{1}$.

Consider the nonlinear map $A: W^{1, p}(\Omega) \rightarrow W^{1, p}(\Omega)^{*}$ defined by

$$
\langle A(u), h\rangle=\int_{\Omega}(a(\nabla u), \nabla h)_{\mathbb{R}^{N}} d z \quad \text { for all } u, h \in W^{1, p}(\Omega) .
$$

From Gasiński-Papageorgiou, ${ }^{13}$ we have the following proposition:

Proposition 2. The map $A(\cdot)$ is bounded (that is, it maps bounded sets to bounded sets), and it is continuous monotone (hence maximal monotone too) and of type $(S)_{+}$which means that " $u_{n} \stackrel{w}{\longrightarrow} u$ in $W^{1, p}(\Omega)$ and $\lim \sup _{n} \rightarrow+\infty\left\langle A\left(u_{n}\right), u_{n}-u\right\rangle \leq 0 \Rightarrow u_{n} \rightarrow u$ in $W^{1, p}(\Omega)$."

We will also need some basic facts about the spectrum of the operator $-\Delta_{p}+\xi(z) I$ with the Robin boundary condition. Recall that $\Delta_{p} u=\operatorname{div}\left(|\nabla u|^{p-2} \nabla u\right)$ for all $u \in W^{1, p}(\Omega)$ (the $p$-Laplace differential operator). So, we consider the following nonlinear eigenvalue problem:

$$
\left\{\begin{array}{cr}
-\Delta_{p} u(z)+\xi(z)|u(z)|^{p-2} u(z)=\widehat{\lambda}|u(z)|^{p-2} u(z) & \text { in } \Omega, \\
\frac{\partial u}{\partial n_{p}}+\beta(z)|u|^{p-2} u=0 & \text { on } \partial \Omega .
\end{array}\right.
$$

In this case, $a(y)=|y|^{p-2} y$ for all $y \in \mathbb{R}^{N}$ and so $\frac{\partial u}{\partial n_{p}}$ is defined by extension of the map

$$
C^{1}(\bar{\Omega}) \ni u \rightarrow \frac{\partial u}{\partial n_{p}}=|\nabla u|^{p-2}(\nabla u, n)_{\mathbb{R}^{N}}=|\nabla u|^{p-2} \frac{\partial u}{\partial n}
$$

By an "eigenvalue," we mean a $\widehat{\lambda} \in \mathbb{R}$ for which problem (4) admits a nontrivial solution $\widehat{u} \in W^{1, p}(\Omega)$ known as an "eigenfunction" corresponding to the eigenvalue $\widehat{\lambda}$. From Ref. 32 (see also Ref. 18), we have that $\widehat{u} \in L^{\infty}(\Omega)$. Then, from the nonlinear regularity theory of Lieberman, ${ }^{22}$ we have that $\widehat{u} \in C^{1}(\bar{\Omega})$. We know that there exists a smallest eigenvalue $\widehat{\lambda}_{1}(\xi, \beta) \in \mathbb{R}$ given by

$$
\widehat{\lambda}_{1}(\xi, \beta)=\inf \left[\frac{\mu(u)}{\|u\|_{p}^{p}}: u \in W^{1, p}(\Omega), u \neq 0\right] .
$$

This eigenvalue has the following properties:

- $\widehat{\lambda}_{1}(\xi, \beta)$ is isolated in the spectrum $\widehat{\sigma}(p)$ of $(4)$ (that is, there exists $\varepsilon>0$ such that $\left.\left.\widehat{\lambda}_{1}(\xi, \beta), \widehat{\lambda}_{1}(\xi, \beta)+\varepsilon\right) \cap \widehat{\sigma}(p)=\emptyset\right)$.

- $\widehat{\lambda}_{1}(\xi, \beta)$ is simple (that is, if $\widehat{u}, \widehat{v}$ are eigenfunctions corresponding to the eigenvalue $\widehat{\lambda}_{1}(\xi, \beta)$, then $\widehat{u}=\eta \widehat{v}$ with $\eta \in \mathbb{R} \backslash\{0\}$ ).

- The eigenfunctions corresponding to $\widehat{\lambda}_{1}(\xi, \beta)$ have constant sign.

Let $\widehat{u}_{1}(\xi, \beta)$ be the $L^{p}$-normalized (that is, $\left.\left\|\widehat{u}_{1}(\xi, \beta)\right\|_{p}=1\right)$ positive eigenfunction, then we have $\widehat{u}_{1}(\xi, \beta) \in C_{+}$. Moreover, from the nonlinear maximum principle (see Ref. 36), we have $\widehat{u}_{1}(\xi, \beta) \in D_{+}$. Since $\widehat{\lambda}_{1}(\xi, \beta)$ is isolated and $\widehat{\sigma}(p) \subseteq \mathbb{R}$ is closed, the second eigenvalue $\widehat{\lambda}_{2}(\xi, \beta)$ is well-defined by $\widehat{\lambda}_{2}(\xi, \beta)=\inf \left[\widehat{\lambda}: \widehat{\lambda} \in \widehat{\sigma}(p), \widehat{\lambda}>\widehat{\lambda}_{1}(\xi, \beta)\right]$.

By the Ljusternik-Schnirelmann minimax scheme, we know that $\widehat{\sigma}(p)$ has a whole strictly increasing sequence $\left\{\widehat{\lambda}_{k}(\xi, \beta)\right\}_{k \in \mathbb{N}} \subseteq \mathbb{R}$ such that $\widehat{\lambda}_{k}(\xi, \beta) \rightarrow+\infty$ as $k \rightarrow+\infty$. These are known as "LS-eigenvalues" or "variational eigenvalues" of (4). We do not know if this sequence exhausts $\widehat{\sigma}(p)$. The Ljusternik-Schnirelmann scheme provides minimax expressions for these eigenvalues. For $\widehat{\lambda}_{2}(\xi, \beta)$, we have an alternative minimax characterization which is more suitable for our purposes. So, let

$$
\partial B_{1}^{L^{p}}=\left\{u \in L^{p}(\Omega):\|u\|_{p}=1\right\}, \quad M=W^{1, p}(\Omega) \cap \partial B_{1}^{L^{p}}
$$

and

$$
\widehat{\Gamma}=\left\{\widehat{\gamma} \in C([-1,1], M): \widehat{\gamma}(-1)=-\widehat{u}_{1}(\xi, \beta), \widehat{\gamma}(1)=\widehat{u}_{1}(\xi, \beta)\right\} .
$$


Proposition 3. $\widehat{\lambda}_{2}(\xi, \beta)=\inf _{\widehat{\gamma} \in \widehat{\Gamma}-1 \leq t \leq 1} \mu(\widehat{\gamma}(t))$.

For more information on these issues, we refer to Refs. 28 and 32. Next, let us recall a few basic definitions and facts from Morse theory (critical groups) which we will need in the sequel. So, let $\left(Y_{1}, Y_{2}\right)$ be a topological pair such that $Y_{2} \subseteq Y_{1} \subseteq X$. For every $k \in \mathbb{N}_{0}$, by $H_{k}\left(Y_{1}, Y_{2}\right)$, we denote the $k$ th-relative singular homology group for the pair $\left(Y_{1}, Y_{2}\right)$ with integer coefficients. If $k \in-\mathbb{N}$, then $H_{k}\left(Y_{1}, Y_{2}\right)=0$. Let $\varphi \in C^{1}(X, \mathbb{R})$ and $c \in \mathbb{R}$. We introduce the following sets:

$$
\begin{gathered}
\varphi^{c}=\{u \in X: \varphi(u) \leq c\}, \quad K_{\varphi}=\left\{u \in X: \varphi^{\prime}(u)=0\right\}, \\
K_{\varphi}^{c}=\left\{u \in K_{\varphi}: \varphi(u)=c\right\} .
\end{gathered}
$$

Suppose that $u_{0} \in K_{\varphi}^{c}$ is isolated. The critical groups of $\varphi$ at $u_{0}$ are defined by

$$
C_{k}\left(\varphi, u_{0}\right)=H_{k}\left(\varphi^{c} \cap U, \varphi^{c} \cap U \backslash\left\{u_{0}\right\}\right) \text { for all } k \in \mathbb{N}_{0},
$$

with $U$ being a neighborhood of $u_{0}$ such that $K_{\varphi} \cap U \cap \varphi^{c}=\left\{u_{0}\right\}$. The excision property of singular homology theory implies that the above definition of critical groups is independent of the choice of the neighborhood $U$. Suppose that $\varphi \in C^{1}(X, \mathbb{R})$ satisfies the $C$ condition and that $\inf \varphi\left(K_{\varphi}\right)>-\infty$. Let $\inf \varphi\left(K_{\varphi}\right)>c$. The critical groups of $\varphi$ at infinity are defined by $C_{k}(\varphi, \infty)=H_{k}\left(X, \varphi^{c}\right)$ for all $k \in \mathbb{N}_{0}$. This definition is independent of the particular choice of the level $c<\inf \varphi\left(K_{\varphi}\right)$. To see this, let $d<c<\inf \varphi\left(K_{\varphi}\right)$. From the noncritical interval theorem (see, for example, Ref. 27, p. 110), we know that $\varphi^{d}$ is a strong deformation retract of $\varphi^{c}$. Therefore, $H_{k}\left(X, \varphi^{c}\right)=H_{k}\left(X, \varphi^{d}\right) \quad$ for all $k \in \mathbb{N}_{0}$. The following proposition is useful in producing additional critical points for a functional $\varphi \in C^{1}(X, \mathbb{R})$ (see Ref. 27):

Proposition 4. If $\varphi \in C^{1}(X, \mathbb{R})$ satisfies the C-condition, for some $k \in \mathbb{N}_{0} C_{k}(\varphi, 0) \neq 0, C_{k}(\varphi, \infty)=0$, then there exists $u \in K_{\varphi}$, $u \neq 0$.

Finally, let us fix our notation. So, for $x \in \mathbb{R}$, we set $x^{ \pm}=\max \{ \pm x, 0\}$ and for $u \in W^{1, p}(\Omega)$ we define $u^{ \pm}(\cdot)=u(\cdot)^{ \pm}$. We know that $u^{ \pm} \in W^{1, p}(\Omega), u=u^{+}-u^{-}$, and $|u|=u^{+}+u^{-}$. By $|\cdot|_{N}$, we denote the Lebesgue measure on $\mathbb{R}^{N}$. If $u, v \in W^{1, p}(\Omega)$, then

$$
[u, v]=\left\{h \in W^{1, p}(\Omega): u(z) \leq h(z) \leq v(z) \text { for a.a. } z \in \Omega\right\} .
$$

For $\Sigma_{0} \subseteq \partial \Omega$ a closed set, we introduce the following set of functions:

$$
\widehat{D}_{+}\left(\Sigma_{0}\right)=\left\{h \in C^{1}(\bar{\Omega}): h(z)>0 \text { for all } z \in \Omega,\left.\frac{\partial h}{\partial n}\right|_{\Sigma_{0}}<0\right\} .
$$

Evidently, this set is open in $C^{1}(\bar{\Omega})$ and we have $D_{+} \subseteq \widehat{D}_{+}\left(\Sigma_{0}\right)$. Note that $\widehat{D}_{+}\left(\Sigma_{0}\right)$ is the interior of the positive (order) cone of $C_{*}^{1}(\bar{\Omega})$ $=\left\{u \in C^{1}(\bar{\Omega}):\left.u\right|_{\Sigma_{0}}=0\right\}$. So, alternatively the analysis below can be done using the pair $C_{*}^{1}(\bar{\Omega}), W_{*}^{1, p}(\Omega)=\overline{C_{*}^{1}(\bar{\Omega})}\|\cdot\|$.

\section{NONHOMOGENEOUS EQUATION}

In this section, we deal with problem (1) and we look for positive solutions. We prove an existence theorem and a multiplicity theorem producing two nontrivial smooth solutions. The two results differ in the geometry of the equation near zero.

For the existence theorem, our hypotheses on the map $a(\cdot)$ are the following:

$H(a)_{1}: a(y)=a_{0}(|y|) y$ for all $y \in \mathbb{R}^{N}$ with $a_{0}(t)>0$ for all $t>0$, and hypotheses $H(a)_{1}$ (i)-(iii) are the same as the corresponding hypotheses $H(a)$ (i)-(iii) and

(iv) if $G_{0}(t)=\int_{0}^{t} a_{0}(s) s d s$, then $p G_{0}(t)-a_{0}(t) t^{2} \geq-\bar{c}$ for all $t>0$ and some $\bar{c}>0$.

Remark 3. As we already mentioned in Sec. II, hypotheses $H(a)_{1}(i)-(i i i)$ come from the nonlinear regularity theory of Lieberman ${ }^{22}$ and the nonlinear maximum principle of Pucci-Serrin ${ }^{36}$ (pp. 110 and 120). Hypothesis $H(a)_{1}($ iv) serves the particular needs of our problem, but it is very mild and it is satisfied in all cases of interest as the examples which follow illustrate.

Example 1. The following maps $a: \mathbb{R}^{N} \rightarrow \mathbb{R}^{N}$ satisfy hypotheses $H(a)_{1}$ :

(a) $\quad a(y)=|y|^{p-2} y$ with $1<p<\infty$. This map corresponds to the $p$-Laplacian defined by $\Delta_{p} u=\operatorname{div}\left(|\nabla u|^{p-2} \nabla u\right)$ for all $u \in W^{1, p}(\Omega)$.

(b) $\quad a(y)=|y|^{p-2} y+|y|^{q-2} y$ with $1<q<p<\infty$. This map corresponds to the $(p, q)$-Laplacian defined by $\Delta_{p} u+\Delta_{q} u$ for all $u \in W^{1, p}(\Omega)$. Such an operator arises in problems of mathematical physics (see, for example, Ref. 5). Recently, there have been some existence and multiplicity results for such equations. We refer to the works of Aizicovici-Papageorgiou-Staicu, ${ }^{2}$ Barile-Figueiredo, ${ }^{3}$ Candito-Livrea-Papageorgiou, ${ }^{4}$ Cingolani-Degiovanni, ${ }^{6}$ Gasiński-Papageorgiou, ${ }^{14,15}$ Marano-Mosconi-Papageorgiou, ${ }^{25}$ PapageorgiouRădulescu, ${ }^{29,31,33,34}$ Sun, ${ }^{38}$ and Sun-Zhang. ${ }^{39}$ 
(c) $a(y)=\left(1+|y|^{2}\right)^{\frac{p-2}{2}} y$ with $1<p<\infty$. This map corresponds to the generalized p-mean curvature differential operator defined by $\operatorname{div}\left(\left(1+|\nabla u|^{2}\right)^{\frac{p-2}{2}} \nabla u\right)$ for all $u \in W^{1, p}(\Omega)$.

(d) $\quad a(y)=|y|^{p-2}\left[1+\frac{1}{1+|y|^{p}}\right]$ with $1<p<\infty$. This map corresponds to the following differential operator $-\Delta_{p} u+\operatorname{div}\left(\frac{|\nabla u|^{p-2} \nabla u}{1+|\nabla u|^{p}}\right)$ for all $u \in W^{1, p}(\Omega)$. This operator arises in problems of plasticity.

Our hypotheses on the reaction term $f(z, x)$ are the following:

$H_{1}: f: \Omega \times \mathbb{R} \rightarrow \mathbb{R}$ is a Carathéodory function such that $f(z, 0)=0$ for a.a. $z \in \Omega$ and

(i) $|f(z, x)| \leq a(z)\left(1+x^{r-1}\right)$ for a.a. $z \in \Omega$, all $x \geq 0$, with $a \in L^{\infty}(\Omega)_{+}, p<r<p^{*}$;

(ii) if $F(z, x)=\int_{0}^{x} f(z, s) d s$, then $\lim _{x \rightarrow+\infty} \frac{F(z, x)}{x^{p}}=+\infty$ uniformly for a.a. $z \in \Omega$;

(iii) if $e(z, x)=f(z, x) x-p F(z, x)$, then there exists $d \in L^{1}(\Omega)$ such that $e(z, x) \leq e(z, y)+d(z)$ for a.a. $z \in \Omega$, all $0 \leq x \leq y$;

(iv) if $\widehat{\xi}=\frac{p-1}{c_{1}} \xi, \widehat{\beta}=\frac{p-1}{c_{1}} \beta$ [see (2)], then there exist functions $\eta, \tilde{\eta} \in L^{\infty}(\Omega)$ such that $\eta(z) \leq \frac{c_{1}}{p-1} \widehat{\lambda_{1}}(\widehat{\xi}, \widehat{\beta})$ for a.a. $z \in \Omega, \eta \neq \frac{c_{1}}{p-1} \widehat{\lambda_{1}}(\widehat{\xi}, \widehat{\beta})$, $\tilde{\eta}(z) \leq \liminf \sin _{x \rightarrow 0^{+}} \frac{f(z, x)}{x^{p-1}} \leq \lim \sup _{x \rightarrow 0^{+}} \frac{f(z, x)}{x^{p-1}} \leq \eta(z)$ uniformly for a.a. $z \in \Omega$.

Remark 4. Since we look for positive solutions and the above hypotheses concern the positive semiaxis, we may assume that $f(z, x)=0$ for a.a. $z \in \Omega$, all $x \leq 0$. Hypotheses $H_{1}$ (ii) and (iii) imply that $\lim _{x \rightarrow+\infty} \frac{f(z, x)}{x^{p-1}}=+\infty$ uniformly for a.a. $z \in \Omega$. So, the reaction term $f(z, \cdot)$ is $(p-1)$ superlinear. However, the superlinearity condition of $f(z, \cdot)$ is not formulated using the so-called Ambrosetti-Rabinowitz condition. We recall that the AR-condition says that there exist $s>p$ and $M>0$ such that

$$
\begin{gathered}
0<s F(z, x) \leq f(z, x) x \text { for a.a. } z \in \Omega \text {, all } x \geq M, \\
0<\operatorname{ess} \inf _{\Omega} F(\cdot, M) .
\end{gathered}
$$

This is a unilateral version of the usual AR-condition since we have assumed that $f(z, 0)=0$ for a.a. $z \in \Omega$, all $x \leq 0$. Integrating (6) and using (6a), we obtain the following weaker condition:

$$
c_{6} x^{s} \leq F(z, x) \quad \text { for a.a. } z \in \Omega \text {, all } x \geq M \text {, some } c_{6}>0 .
$$

From (4), we see that, for a.a. $z \in \Omega, F(z$, ) eventually has $s$-polynomial growth. From (6), this implies that for a.a. $z \in \Omega, f(z, \cdot)$ eventually has $(s-1)$-polynomial growth. This excludes from consideration $(p-1)$-superlinear nonlinearities with slower growth near $+\infty$ (see the examples below). Here, instead of (6) and (6a), we use hypothesis $H_{1}$ (iii) which includes such nonlinearities. Hypothesis $H_{1}($ iii) is a quasimonotonicity condition on the function $e(z, \cdot)$. This condition is a little more general than the one used by Li-Yang. ${ }^{24}$ Hypothesis $H_{1}$ (iii) is satisfied if there exists $M>0$ such that for a.a. $z \in \Omega$, the function $x \rightarrow \frac{f(z, x)}{x^{p-1}}$ is nondecreasing on $[M,+\infty)$ or if for a.a. $z \in \Omega$, the function $x \rightarrow e(z, x)$ is nondecreasing on $[M,+\infty)$.

Example 2. The following functions satisfy hypotheses $H_{1}$. For the sake of simplicity, we drop the $z$-dependence,

$$
f_{1}(x)= \begin{cases}9 x^{p-1} & \text { if } x \in[0,1], \\ x^{r-1}+(9-1) x^{\tau-1} & \text { if } x>1,\end{cases}
$$

with $\vartheta<\widehat{\lambda}_{1}(\widehat{\xi}, \widehat{\beta})$ and $p, \tau<r$. Also, $f_{2}(x)=x^{p-1}[\ln (1+x)+\vartheta]$ with $\vartheta<\widehat{\lambda}_{1}(\widehat{\xi}, \widehat{\beta})$. Note that $f_{2}$ does not satisfy the AR-condition.

Let $\vartheta>\|\xi\|_{\infty}$ [see hypothesis $\left.H(\xi)\right]$. We introduce the following Carathéodory function:

$$
\widehat{f}(z, x)=f(z, x)+\vartheta\left(x^{+}\right)^{p-1}= \begin{cases}0 & \text { if } x \leq 0 \\ f(z, x)+\vartheta x^{p-1} & \text { if } 0<x .\end{cases}
$$

We set $\widehat{F}(z, x)=\int_{0}^{x} \widehat{f}(z, s) d s$ and consider the $C^{1}$-functional $\widehat{\varphi}: W^{1, p}(\Omega) \rightarrow \mathbb{R}$ defined by

$$
\widehat{\varphi}(u)=\frac{1}{p} \mu(u)+\frac{9}{p}\|u\|_{p}^{p}-\int_{\Omega} \widehat{F}(z, u) d z \quad \text { for all } u \in W^{1, p}(\Omega) .
$$


Proposition 5. If hypotheses $H(a)_{1}, H(\xi), H(\beta)$, and $H_{1}(i)$-(iii) hold, then $\widehat{\varphi}$ satisfies the $C$-condition.

Proof. Let $\left\{u_{n}\right\}_{n \in \mathbb{N}} \subseteq W^{1, p}(\Omega)$ be a sequence such that

$$
\begin{gathered}
\left|\widehat{\varphi}\left(u_{n}\right)\right| \leq M_{1} \quad \text { for some } M_{1}>0, \text { all } n \in \mathbb{N}, \\
\left(1+\left\|u_{n}\right\|\right) \varphi^{\prime}\left(u_{n}\right) \rightarrow 0 \text { in } W^{1, p}(\Omega)^{*} \text { as } n \rightarrow+\infty .
\end{gathered}
$$

From (10), we have

$$
\begin{array}{r}
\left.\left|\left\langle A\left(u_{n}\right), h\right\rangle+\int_{\Omega}(\xi(z)+\vartheta)\right| u_{n}\right|^{p-2} u_{n} h d z \\
+\int_{\partial \Omega} \beta(z)\left|u_{n}\right|^{p-2} u_{n} h d \sigma-\int_{\Omega} \widehat{f}\left(z, u_{n}\right) h d z \mid \\
\leq \frac{\varepsilon_{n}\|h\|}{1+\left\|u_{n}\right\|}, \quad \text { for all } h \in W^{1, p}(\Omega) \text { with } \varepsilon_{n} \rightarrow 0^{+} .
\end{array}
$$

In (11), we choose $h=-u_{n}^{-} \in W^{1, p}(\Omega)$. Then,

$$
\begin{aligned}
& \frac{c_{0}}{p-1}\left\|\nabla u_{n}^{-}\right\|_{p}^{p}+\int_{\Omega}(\xi(z)+\vartheta)\left(u_{n}^{-}\right)^{p} d z \leq \varepsilon_{n} \text { for all } n \in \mathbb{N} \\
& {[\text { see Lemma 1, hypothesis } H(\beta) \text { and }(8)] } \\
\Rightarrow & \left.u_{n}^{-} \rightarrow 0 \text { in } W^{1, p}(\Omega) \text { as } n \rightarrow+\infty \text { (recall that } \vartheta>\|\xi\|_{\infty}\right) .
\end{aligned}
$$

From (9) and (12), we have

$$
\begin{array}{r}
\int_{\Omega} p G\left(\nabla u_{n}^{+}\right) d z+\int_{\Omega}(\xi(z)+\vartheta)\left(u_{n}^{+}\right)^{p} d z+\int_{\partial \Omega} \beta(z)\left(u_{n}^{+}\right)^{p} d \sigma \\
-\int_{\Omega} p \widehat{F}\left(z, u_{n}^{+}\right) d z \leq M_{2}
\end{array}
$$

for some $M_{2}>0$, all $n \in \mathbb{N}$.

In (11), we choose $h=u_{n}^{+} \in W^{1, p}(\Omega)$. Then,

$$
\begin{array}{r}
-\int_{\Omega}\left(a\left(\nabla u_{n}^{+}\right), \nabla u_{n}^{+}\right)_{\mathbb{R}^{N}} d z-\int_{\Omega}(\xi(z)+\vartheta)\left(u_{n}^{+}\right)^{p} d z \\
-\int_{\partial \Omega} \beta(z)\left(u_{n}^{+}\right)^{p} d \sigma+\int_{\Omega} \widehat{f}\left(z, u_{n}^{+}\right) u_{n}^{+} d z \leq \varepsilon_{n},
\end{array}
$$

for all $n \in \mathbb{N}$. Adding (13) and (14) and using hypothesis $H(a)_{1}$ (iv), we obtain

$$
\int_{\Omega} e\left(z, u_{n}^{+}\right) d z \leq M_{3} \quad \text { for some } M_{3}>0 \text {, all } n \in \mathbb{N} .
$$

Claim: $\left\{u_{n}^{+}\right\}_{n \in \mathbb{N}} \subseteq W^{1, p}(\Omega)$ is bounded.

We argue by contradiction. So, suppose that the claim is not true. By passing to a subsequence if necessary, we may assume that

$$
\left\|u_{n}^{+}\right\| \rightarrow+\infty \quad \text { as } n \rightarrow+\infty .
$$

Let $y_{n}=\frac{u_{n}^{+}}{\left\|u_{n}^{+}\right\|}, n \in \mathbb{N}$. Then, $\left\|y_{n}\right\|=1, y_{n} \geq 0$ for all $n \in \mathbb{N}$. So, we may assume that

$$
y_{n} \stackrel{w}{\rightarrow} y \text { in } W^{1, p}(\Omega) \text { and } y_{n} \rightarrow y \text { in } L^{r}(\Omega) \text { and in } L^{p}(\partial \Omega), y \geq 0 .
$$

First, assume that $y \neq 0$ and let $\Omega_{+}=\{z \in \Omega: y(z)>0\}$ \{recall that $y \geq 0$ [see (17)]\}. Then, $\left|\Omega_{+}\right|_{N}>0$ and we have

$$
u_{n}^{+}(z) \rightarrow+\infty \quad \text { for a.a. } z \in \Omega_{+} .
$$

From (18) and hypothesis $H_{1}(\mathrm{ii})$, it follows that 


$$
\frac{F\left(z, u_{n}^{+}(z)\right)}{u_{n}^{+}(z)^{p}} \rightarrow+\infty \quad \text { for a.a. } z \in \Omega_{+}
$$

Using (19), we have

$$
\lim _{n \rightarrow+\infty} \frac{F\left(z, u_{n}^{+}(z)\right)}{\left\|u_{n}^{+}\right\|^{p}}=\lim _{n \rightarrow+\infty} \frac{F\left(z, u_{n}^{+}(z)\right)}{u_{n}^{+}(z)^{p}} y_{n}(z)^{p}=+\infty
$$

for a.a. $z \in \Omega_{+}$. Then, invoking Fatou's lemma [hypotheses $H_{1}$ (i) and (ii) permit its use], we obtain

$$
\lim _{n \rightarrow+\infty} \int_{\Omega_{+}} \frac{F\left(z, u_{n}^{+}\right)}{\left\|u_{n}^{+}\right\|^{p}} d z=+\infty
$$

Hypothesis $H_{1}$ (ii) implies that we can find $M_{4}>0$ such that

$$
F(z, x) \geq 0 \quad \text { for a.a. } z \in \Omega \text {, all } x \geq M_{4} .
$$

Then,

$$
\begin{aligned}
\int_{\Omega \backslash \Omega_{+}} \frac{F\left(z, u_{n}^{+}\right)}{\left\|u_{n}^{+}\right\|^{p}} d z & =\int_{\left(\Omega \backslash \Omega_{+}\right) \cap\left\{u_{n}^{+} \geq M_{4}\right\}} \frac{F\left(z, u_{n}^{+}\right)}{\left\|u_{n}^{+}\right\|^{p}} d z \\
& +\int_{\left(\Omega \backslash \Omega_{+}\right) \cap\left\{u_{n}^{+}<M_{4}\right\}} \frac{F\left(z, u_{n}^{+}\right)}{\left\|u_{n}^{+}\right\|^{p}} d z \geq-c_{7}
\end{aligned}
$$

for all $n \in \mathbb{N}$, some $c_{7}>0$ [see (21) and use hypothesis $\left.H_{1}(\mathrm{i})\right]$. Hence, we have

$$
\begin{aligned}
& \int_{\Omega} \frac{F\left(z, u_{n}^{+}\right)}{\left\|u_{n}^{+}\right\|^{p}} d z=\int_{\Omega_{+}} \frac{F\left(z, u_{n}^{+}\right)}{\left\|u_{n}^{+}\right\|^{p}} d z+\int_{\Omega \backslash \Omega_{+}} \frac{F\left(z, u_{n}^{+}\right)}{\left\|u_{n}^{+}\right\|^{p}} d z \\
& \geq \int_{\Omega_{+}} \frac{F\left(z, u_{n}^{+}\right)}{\left\|u_{n}^{+}\right\|^{p}} d z-c_{7} \quad \text { for all } n \in \mathbb{N}[\operatorname{see}(22)] \\
\Rightarrow & \lim _{n \rightarrow+\infty} \int_{\Omega} \frac{F\left(z, u_{n}^{+}\right)}{\left\|u_{n}^{+}\right\|^{p}} d z=+\infty \quad[\operatorname{see}(20)] .
\end{aligned}
$$

From (9) and (12), we have

$$
\begin{aligned}
& \int_{\Omega} \widehat{F}\left(z, u_{n}^{+}\right) d z \leq M_{5}+\int_{\Omega} G\left(\nabla u_{n}^{+}\right) d z \\
+ & \frac{1}{p} \int_{\Omega}(\xi(z)+9)\left(u_{n}^{+}\right)^{p} d z+\frac{1}{p} \int_{\partial \Omega} \beta(z)\left(u_{n}^{+}\right)^{p} d \sigma, \\
& \text { for some } M_{5}>0, \text { all } n \in \mathbb{N}, \\
\Rightarrow \quad & \int_{\Omega} \frac{\widehat{F}\left(z, u_{n}^{+}\right)}{\left\|u_{n}^{+}\right\| p} d z \leq \frac{M_{5}}{\left\|u_{n}^{+}\right\|^{p}}+c_{5}\left(\frac{1}{\left\|u_{n}^{+}\right\|^{p}}+\|\nabla y\|_{p}^{p}\right) \\
+ & \frac{1}{p} \int_{\Omega}(\xi(z)+9) y_{n}^{p} d z+\frac{1}{p} \int_{\partial \Omega} \beta(z) y_{n}^{p} d \sigma, \\
& \quad \text { for all } n \in \mathbb{N}(\text { see Corollary } 1) \\
\Rightarrow \quad & \int_{\Omega} \frac{\widehat{F}\left(z, u_{n}^{+}\right)}{\left\|u_{n}^{+}\right\|^{p}} d z \leq M_{6} \quad \text { for some } M_{6}>0, \text { all } n \in \mathbb{N} .
\end{aligned}
$$

Comparing (23) and (24), we have a contradiction. Next, we assume that $y=0$. For $k>0$, we set $v_{n}=k^{\frac{1}{p}} y_{n}$ for all $n \in \mathbb{N}$. Evidently, we have

$$
v_{n} \rightarrow 0 \text { in } L^{r}(\Omega) \quad[\text { see (17) and recall that } y=0] .
$$

Hypothesis $H_{1}(\mathrm{i})$ and Krasnoselskii's theorem (see, for example, Ref. 12, p. 407) imply that

$$
\int_{\Omega} F\left(z, v_{n}\right) d z \rightarrow 0 \quad \text { as } n \rightarrow+\infty \quad[\operatorname{see}(25)] .
$$

Because of (16), we see that we can find $n_{0} \in \mathbb{N}$ such that 


$$
0<k^{\frac{1}{p}} \frac{1}{\left\|u_{n}^{+}\right\|^{p}} \leq 1 \quad \text { for all } n \geq n_{0}
$$

Consider the $C^{1}$-functional $\widehat{\varphi}_{0}: W^{1, p}(\Omega) \rightarrow \mathbb{R}$ defined by

$$
\widehat{\varphi}_{0}(u)=\frac{c_{1}}{p(p-1)}\|\nabla u\|_{p}^{p}+\frac{1}{p} \int_{\Omega}(\xi(z)+\vartheta)|u|^{p} d z+\frac{1}{p} \int_{\partial \Omega} \beta(z)|u|^{p} d \sigma-\int_{\Omega} \widehat{F}(z, u) d z
$$

for all $u \in W^{1, p}(\Omega)$. Let $t_{n} \in[0,1]$ be such that

$$
\widehat{\varphi}_{0}\left(t_{n} u_{n}^{+}\right)=\max \left[\widehat{\varphi}_{0}\left(t u_{n}^{+}\right): 0 \leq t \leq 1\right] \quad \text { for all } n \in \mathbb{N} .
$$

On account of (27) and (28), we have

$$
\begin{aligned}
& \widehat{\varphi}_{0}\left(t_{n} u_{n}^{+}\right) \geq \widehat{\varphi}_{0}\left(v_{n}\right) \\
&=\frac{c_{1}}{p(p-1)}\left\|\nabla v_{n}\right\|_{p}^{p}+\frac{1}{p} \int_{\Omega} \xi(z) v_{n}^{p} d z \\
& \quad+\frac{1}{p} \int_{\partial \Omega} \beta(z) v_{n}^{p} d \sigma-\int_{\Omega} F\left(z, v_{n}\right) d z \quad \text { [see (8)] } \\
& \geq \frac{1}{p}\left[\frac{c_{1}}{(p-1)}\left\|\nabla v_{n}\right\|_{p}^{p}+\int_{\Omega}(\xi(z)+\vartheta) v_{n}^{p} d z\right] \\
&-\frac{\vartheta}{p}\left\|v_{n}\right\|_{p}^{p}-\int_{\Omega} F\left(z, v_{n}\right) d z \\
&\left(\text { see hypothesis } H(\beta), \text { recall that } \vartheta>\|\xi\|_{\infty}\right) \\
& \geq c_{8}\left\|v_{n}\right\|^{p}-\frac{\vartheta}{p}\left\|v_{n}\right\|_{p}^{p}-\int_{\Omega} F\left(z, v_{n}\right) d z \\
& \text { for all } n \geq n_{0}, \text { some } c_{8}>0, \\
& \geq c_{8} k-\frac{\vartheta}{p}\left\|v_{n}\right\|_{p}^{p}-\int_{\Omega} F\left(z, v_{n}\right) d z \quad \text { for all } n \geq n_{0} \\
& \quad\left(\text { recall that }\left\|y_{n}\right\|=1 \text { for all } n \in \mathbb{N}\right) \\
& \geq \frac{c_{8}}{2} k \quad \text { for all } n \geq n_{1} \geq n_{0} \quad[\text { see }(25) \text { and (26) }] .
\end{aligned}
$$

However, $k>0$ is arbitrary. So, we infer that

$$
\widehat{\varphi}_{0}\left(t_{n} u_{n}^{+}\right) \rightarrow+\infty \quad \text { as } n \rightarrow+\infty \text {. }
$$

Note that

$$
\widehat{\varphi}_{0}(0)=0 \quad \text { and } \quad \widehat{\varphi}_{0}\left(u_{n}^{+}\right) \leq M_{1} \text { for all } n \in \mathbb{N}
$$

[see (9) and note that $\widehat{\varphi}_{0}\left(u_{n}^{+}\right) \leq \widehat{\varphi}_{0}\left(u_{n}\right) \leq \widehat{\varphi}\left(u_{n}\right)$ for all $n \in \mathbb{N}$ ].

Then, (29) and (30) imply that

$$
\begin{aligned}
& t_{n} \in(0,1) \quad \text { for all } n \geq n_{2} \\
\Rightarrow \quad & \left.\frac{d}{d t} \widehat{\varphi}_{0}\left(t u_{n}^{+}\right)\right|_{t=t_{n}}=0 \quad \text { for all } n \geq n_{2} \quad \text { [see (28)] } \\
\Rightarrow \quad & \left\langle\widehat{\varphi}_{0}^{\prime}\left(t_{n} u_{n}^{+}\right), t_{n} u_{n}^{+}\right\rangle=0 \quad \text { (by the chain rule) } \\
\Rightarrow \quad & \frac{c_{0}}{p-1}\left\|\nabla\left(t_{n} u_{n}^{+}\right)\right\|_{p}^{p}+\int_{\Omega} \xi(z)\left(t_{n} u_{n}^{+}\right)^{p} d z \\
& +\int_{\partial \Omega} \beta(z)\left(t_{n} u_{n}^{+}\right)^{p} d \sigma=\int_{\Omega} \widehat{f}\left(z, t_{n} u_{n}^{+}\right)\left(t_{n} u_{n}^{+}\right) d z, \\
\Rightarrow \quad & p \widehat{\varphi}_{0}\left(t_{n} u_{n}^{+}\right)=\int_{\Omega} e\left(z, t_{n} u_{n}^{+}\right) d z \leq \int_{\Omega} e\left(z, u_{n}^{+}\right) d z+\|d\|_{1} \\
& \quad \text { for all } n \geq n_{2}\left[\text { see } H_{1}(\text { iii) }]\right. \\
\Rightarrow \quad & p \widehat{\varphi}_{0}\left(t_{n} u_{n}^{+}\right) \leq M_{7} \text { for some } M_{7} \geq 0, \text { all } n \geq n_{2}[\text { see (15)]. }
\end{aligned}
$$


Comparing (29) and (31), we have a contradiction. This proves the claim. The claim and (12) imply that $\left\{u_{n}\right\}_{n \in \mathbb{N}} \subseteq W^{1, p}(\Omega)$ is bounded. So, we may assume that

$$
u_{n} \stackrel{w}{\rightarrow} u \text { in } W^{1, p}(\Omega) \text { and } u_{n} \rightarrow u \text { in } L^{r}(\Omega) \text { and in } L^{p}(\partial \Omega) .
$$

In (11), we choose $h=u_{n}-u \in W^{1, p}(\Omega)$, pass to the limit as $n \rightarrow+\infty$, and use (32). Then,

$$
\begin{aligned}
& \lim _{n \rightarrow+\infty}\left\langle A\left(u_{n}\right), u_{n}-u\right\rangle=0 \\
\Rightarrow & u_{n} \rightarrow u \text { in } W^{1, p}(\Omega) \\
\Rightarrow & \widehat{\varphi} \text { satisfies the } C-\text { condition. }
\end{aligned}
$$

To proceed further, we will need Lemma 2. This lemma will help us to establish the geometry near the origin.

Lemma 2. If $\eta \in L^{\infty}(\Omega)$ is as in hypothesis $H_{1}(i v)$, then we can find $c_{9}>0$ such that

$$
\begin{aligned}
& \frac{c_{1}}{p-1}\|\nabla u\|_{p}^{p}+\int_{\Omega}(\xi(z)-\eta(z))|u|^{p} d z+\int_{\partial \Omega} \beta(z)|u|^{p} d \sigma \\
& \geq c_{9}\|u\|^{p} \quad \text { for all } u \in W^{1, p}(\Omega) .
\end{aligned}
$$

Proof. We have

$$
\frac{c_{1}}{p-1}\left[\|\nabla u\|_{p}^{p}+\int_{\Omega}(\widehat{\xi}(z)-\widehat{\eta}(z))|u|^{p} d z+\int_{\partial \Omega} \widehat{\beta}(z)|u|^{p} d \sigma\right]
$$

with $\widehat{\xi}=\frac{p-1}{c_{1}} \xi, \widehat{\beta}=\frac{p-1}{c_{1}} \beta, \widehat{\eta}=\frac{p-1}{c_{1}} \eta$ [see also hypothesis $\left.H_{1}(\mathrm{iv})\right]$.

Let $\widehat{\psi}: W^{1, p}(\Omega) \rightarrow \mathbb{R}$ be the $C^{1}$-functional defined by

$$
\widehat{\psi}(u)=\|\nabla u\|_{p}^{p}+\int_{\Omega}(\widehat{\xi}(z)-\widehat{\eta}(z))|u|^{p} d z+\int_{\partial \Omega} \widehat{\beta}(z)|u|^{p} d \sigma
$$

for all $u \in W^{1, p}(\Omega)$. We have

$$
\begin{gathered}
\widehat{\psi}(u) \geq \int_{\Omega}\left[\widehat{\lambda}_{1}(\widehat{\xi}, \widehat{\beta})-\eta(z)\right]|u|^{p} d z \geq 0 \\
{\left[\text { see (5) and hypothesis } H_{1}(\mathrm{iv})\right] .}
\end{gathered}
$$

Evidently, it is enough to prove the lemma for $\widehat{\psi}$. Arguing indirectly, and since $\widehat{\psi}$ is $p$-homogeneous, suppose we can find $\left\{u_{n}\right\}_{n \in \mathbb{N}} \subseteq W^{1, p}(\Omega)$ such that

$$
\left\|u_{n}\right\|=1 \text { for all } n \in \mathbb{N} \text { and } \widehat{\psi}\left(u_{n}\right) \downarrow 0 \text { as } n \rightarrow+\infty \text {. }
$$

We may assume that

$$
u_{n} \stackrel{w}{\longrightarrow} u \text { in } W^{1, p}(\Omega) \text { and } u_{n} \rightarrow u \text { in } L^{p}(\Omega) \text { and in } L^{p}(\partial \Omega) .
$$

The functional $\widehat{\psi}(\cdot)$ is sequentially weakly lower semicontinuous. So, from (33) and (34), it follows that

$$
\begin{aligned}
& \widehat{\psi}(u) \leq 0, \\
& \|\nabla u\|_{p}^{p}+\int_{\Omega} \widehat{\xi}(z)|u|^{p} d z+\int_{\partial \Omega} \widehat{\beta}(z)|u|^{p} d \sigma \leq \int_{\Omega} \widehat{\eta}(z)|u|^{p} d z \\
\Rightarrow & \mu(u) \leq \int_{\Omega} \widehat{\eta}(z)|u|^{p} d z \leq \widehat{\lambda}_{1}(\widehat{\xi}, \widehat{\beta})\|u\|_{p}^{p} \\
\Rightarrow & u=\lambda \widehat{u}_{1}(\widehat{\xi}, \widehat{\beta}) \quad \text { for some } \lambda \in \mathbb{R} \quad[\operatorname{see}(5)] .
\end{aligned}
$$

If $\lambda=0$, then $u=0$ and so $u_{n} \rightarrow 0$ in $W^{1, p}(\Omega)$, a contradiction to the fact that $\left\|u_{n}\right\|=1$ for all $n \in \mathbb{N}$. If $\lambda \neq 0$, then to fix things we assume that $\lambda>0$ (the reasoning is similar if $\lambda<0$ ). We have $u=\lambda \widehat{u}_{1}(\widehat{\xi}, \widehat{\beta}) \in D_{+}$. So, from (35) and the hypothesis on $\eta$ [see hypothesis $H_{1}$ (iv)], we obtain $\mu(u) \leq \widehat{\lambda}_{1}(\widehat{\xi}, \widehat{\beta})\|u\|_{p}^{p}$, which contradicts (5).

Using this lemma, we can determine the geometry near zero for $\widehat{\varphi}$. This is the first step in establishing the mountain pass geometry for $\widehat{\varphi}$. 
Proposition 6. If hypotheses $H(a)_{1}, H(\xi), H(\beta)$, and $H_{1}$ hold, then $u=0$ is a local minimizer of $\widehat{\varphi}$.

Proof. Hypotheses $H_{1}(\mathrm{i})$, (iv) imply that given $\varepsilon>0$, we can find $c_{10}=c_{10}(\varepsilon)>0$ such that

$$
F(z, x) \leq \frac{1}{p}(\eta(z)+\varepsilon) x^{p}+c_{10} x^{r} \quad \text { for a.a. } z \in \Omega \text {, all } x \geq 0 .
$$

Then, for $u \in W^{1, p}(\Omega)$, we have

$$
\begin{aligned}
\widehat{\varphi}(u) & \int_{\Omega} G(\nabla u) d z+\frac{1}{p} \int_{\Omega}(\xi(z)+\mu)|u|^{p} d z+\frac{1}{p} \int_{\partial \Omega} \beta(z)|u|^{p} d \sigma \\
& -\int_{\Omega} \widehat{F}(z, u) d z \\
\geq & \frac{1}{p}\left[\frac{c_{1}}{p-1}\|\nabla u\|_{p}^{p}+\int_{\Omega} \xi(z)|u|^{p} d z+\int_{\partial \Omega} \beta(z)|u|^{p} d \sigma\right] \\
& -\int_{\Omega} F\left(z, u^{+}\right) d z \quad[\text { see Corollary } 1 \text { and }(8)] \\
\geq & \frac{1}{p}\left[\frac{c_{1}}{p-1}\|\nabla u\|_{p}^{p}+\int_{\Omega}(\xi(z)-\eta(z))|u|^{p} d z+\int_{\partial \Omega} \beta(z)|u|^{p} d \sigma\right] \\
- & \frac{\varepsilon}{p}\|u\|^{p}-c_{11}\|u\|^{r} \quad \text { for some } c_{11}>0[\text { see (36)] } \\
\geq & \frac{1}{p}\left[c_{9}-\varepsilon\right]\|u\|^{p}-c_{11}\|u\|^{r} \quad(\text { see Lemma 2). }
\end{aligned}
$$

Choosing $\varepsilon \in\left(0, c_{9}\right)$, from (37), we infer that

$$
\widehat{\varphi}(u) \geq c_{12}\|u\|^{p}-c_{11}\|u\|^{r} \quad \text { for all } u \in W^{1, p}(\Omega) \text {, some } c_{12}>0
$$

Since $p<r$, we see that we can find $\rho \in(0,1)$ small such that

$$
\begin{aligned}
& \widehat{\varphi}(u)>0=\widehat{\varphi}(0) \quad \text { for all } u \in W^{1, p}(\Omega), 0<\|u\| \leq \rho \\
\Rightarrow \quad & u=0 \text { is a (strict) local minimizer of } \widehat{\varphi} .
\end{aligned}
$$

As a consequence of hypothesis $H_{1}$ (ii), we have the following proposition:

Proposition 7. If hypotheses $H(a)_{1}, H(\xi), H(\beta)$, and $H_{1}$ hold and $u \in D_{+}$, then $\widehat{\varphi}(t u) \rightarrow-\infty$ as $t \rightarrow+\infty$.

Proposition 8. If hypotheses $H(a)_{1}, H(\xi), H(\beta)$, and $H_{1}$ hold, then $K_{\widehat{\varphi}} \subseteq C_{+}$.

Proof. Let $u_{0} \in K_{\widehat{\varphi}}$. Then,

$$
\begin{aligned}
& \widehat{\varphi}^{\prime}\left(u_{0}\right)=0 \\
\Rightarrow \quad & \left\langle A\left(u_{0}\right), h\right\rangle+\int_{\Omega}(\xi(z)+9)\left|u_{0}\right|^{p-2} u_{0} h d z \\
& +\int_{\partial \Omega} \beta(z)\left|u_{0}\right|^{p-2} u_{0} h d \sigma=\int_{\Omega} \widehat{f}\left(z, u_{0}\right) h d z
\end{aligned}
$$

for all $h \in W^{1, p}(\Omega)$. In (38), we choose $h=-u_{0}^{-} \in W^{1, p}(\Omega)$, and using Lemma 1 , we have

$$
\begin{aligned}
& \frac{c_{1}}{p-1}\left\|\nabla u_{0}^{-}\right\|_{p}^{p}+\int_{\Omega}(\xi(z)+\vartheta)\left(u_{0}^{-}\right)^{p} d z \leq 0 \\
& {[\text { see }(8) \text { and hypothesis } H(\beta)] } \\
\Rightarrow & c_{13}\left\|u_{0}^{-}\right\|^{p} \leq 0 \quad \text { for some } c_{13}>0 \quad\left(\text { recall } \vartheta>\|\xi\|_{\infty}\right) \\
\Rightarrow & u_{0} \geq 0 .
\end{aligned}
$$


So, we have the following equation (see Ref. 30):

$$
\begin{aligned}
& \left\langle A\left(u_{0}\right), h\right\rangle+\int_{\Omega} \xi(z) u_{0}^{p-1} h d z+\int_{\partial \Omega} \beta(z) u_{0}^{p-1} h d \sigma \\
& =\int_{\Omega} f\left(z, u_{0}\right) h d z \text { for all } h \in W^{1, p}(\Omega) \\
\Rightarrow & \begin{cases}-\operatorname{div} a\left(\nabla u_{0}(z)\right)+\xi(z) u_{0}(z)^{p-1}=f\left(z, u_{0}(z)\right) \\
\frac{\partial u_{0}}{\partial n_{a}}+\beta(z) u_{0}^{p-1} u=0 \text { on } \partial \Omega & \text { for a.a. } z \in \Omega,\end{cases}
\end{aligned}
$$

(see Ref. 30). Now, we can use Proposition 2.10 of Ref. 32 (see also Ref. 18) on (39) and have that

$$
u_{0} \in L^{\infty}(\Omega)
$$

Then, (40) and the nonlinear regularity theory of Lieberman ${ }^{22}$ imply that

$$
\begin{aligned}
& u_{0} \in C_{+} \\
\Rightarrow \quad & K_{\widehat{\varphi}} \subseteq C_{+} .
\end{aligned}
$$

In what follows, we assume that $K_{\widehat{p}}$ is finite. Otherwise, we already have infinitely many positive smooth solutions for problem (1) (see Proposition 8). Now, we are ready to produce positive solutions.

Theorem 2. If hypotheses $H(a)_{1}, H(\xi), H(\beta)$, and $H_{1}$ hold, then problem (1) admits at least one positive solution $u_{0} \in D_{+}$.

Proof. Proposition 6 and since $K_{\widehat{\varphi}}$ is finite imply that we can find $\rho \in(0,1)$ small such that

$$
\widehat{\varphi}(0)=0<\inf [\widehat{\varphi}(u):\|u\|=\rho]=\widehat{m}_{\rho}
$$

(see Ref. 1, Proof of Proposition 29). Combining (41) with Propositions 5 and 7, we see that we can apply Theorem 1 (the mountain pass theorem) and find $u_{0} \in W^{1, p}(\Omega)$ such that

$$
u_{0} \in K_{\widehat{\varphi}} \quad \text { and } \quad \widehat{m}_{\rho} \leq \widehat{\varphi}\left(u_{0}\right)
$$

From (41), (42), and Proposition 8, we infer that $u_{0} \in C_{+} \backslash\{0\}$. Let $\rho=\left\|u_{0}\right\|_{\infty}$. Hypotheses $H_{1}(\mathrm{i})$, (iv) imply that we can find $\widehat{\xi}_{\rho}>0$ such that $f(z, x)+\widehat{\xi}_{\rho} x^{p-1} \geq 0$ for a.a. $z \in \Omega$, all $x \in[0, \rho]$. Using this in (39), we obtain the following equation (see Ref. 36, p. 120):

$$
\begin{array}{ll} 
& \operatorname{div} a\left(\nabla u_{0}(z)\right) \leq\left[\|\xi\|_{\infty}+\widehat{\xi}_{\rho}\right] u_{0}(z)^{p-1} \quad \text { for a.a. } z \in \Omega, \\
\Rightarrow & u_{0} \in D_{+} .
\end{array}
$$

By changing the geometry of the problem near zero, we can have a multiplicity theorem for the positive solutions of (1). So, we strengthen a little the conditions on $a(\cdot)$ and modify the hypotheses on the reaction term $f(z, \cdot)$. The new hypotheses on the map $y \rightarrow a(y)$ are the following:

$H(a)_{2}: a(y)=a_{0}(|y|) y$ for all $y \in \mathbb{R}^{N}$ with $a_{0}(t)>0$ for all $t>0$, and hypotheses $H(a)_{2}(\mathrm{i})$-(iii) are the same as the corresponding hypotheses $H(a)$ (i)-(iii) and

(iv) if $G_{0}(t)=\int_{0}^{t} a_{0}(s) s d s$, then $p G_{0}(t)-a_{0}(t) t^{2} \geq-\bar{c}$ for all $t \geq 0$, some $\bar{c}>0$, and there exists $q \in(1, p]$ such that lim $\sup _{t \rightarrow 0^{+}} \frac{G_{0}(t)}{t^{q}}<+\infty$ and $t \rightarrow G_{0}\left(t^{1 / q}\right)$ is convex.

Remark 5. All the examples given after hypotheses $H(a)_{1}$ also satisfy the new conditions $H(a)_{2}$.

The new hypotheses on the reaction term $f(z, x)$ are the following:

$H_{2}: f: \Omega \times \mathbb{R} \rightarrow \mathbb{R}$ is a Carathéodory function such that $f(z, 0)=0$ for a.a. $z \in \Omega$, hypotheses $H_{2}$ (i)-(iii) are the same as the corresponding hypotheses $H_{1}$ (i)-(iii) and

(iv) with $q \in(1, p]$ as in hypothesis $H(a)_{2}$ (iv), we have $\lim _{x \rightarrow 0^{+}} \frac{f(z, x)}{x^{q-1}}=+\infty$ uniformly for a.a. $z \in \Omega$;

(v) there exists $w_{+} \in C^{1}(\bar{\Omega})$ such that 


$$
\begin{aligned}
& w_{+}(z) \geq c_{+}>0 \quad \text { for all } z \in \bar{\Omega}, \\
& f\left(z, w_{+}(z)\right)-\xi(z) w_{+}(z)^{p-1} \leq-c_{14}<0 \quad \text { for a.a. } z \in \Omega, \\
& \operatorname{div} a\left(\nabla w_{+}\right) \in L^{r^{\prime}}(\Omega) \quad\left(\frac{1}{r}+\frac{1}{r^{\prime}}=1\right), \quad 0 \leq-\operatorname{div} a\left(\nabla w_{+}(z)\right) \\
& \quad \text { for a.a. } z \in \Omega,
\end{aligned}
$$

(vi) there exists $\widehat{\xi}_{+}>0$ such that for a.a. $z \in \Omega$ the function $x \rightarrow f(z, x)+\widehat{\xi}_{+} x^{p-1}$ is nondecreasing on $\left[0,\left\|w_{+}\right\|_{\infty}\right]$.

Remark 6. Hypothesis $\mathrm{H}_{2}$ (iv) implies the presence of a concave term near the origin. If $\xi(z) \geq 0$ for a.a. $z \in \Omega$ and $f\left(z, c_{+}\right) \leq 0$ for a.a. $z \in \Omega$, then $w_{+} \equiv c_{+}$satisfies hypothesis $\mathrm{H}_{2}(\mathrm{v})$.

Theorem 3. If hypotheses $H(a)_{2}, H(\xi), H(\beta)$, and $H_{2}$ hold, then problem (1) has at least two positive solutions $u_{0}$, $\widehat{u} \in D_{+}$and $u_{0} \leq \widehat{u}$, $u_{0} \neq \widehat{u}$.

Proof. As before, let $\vartheta>\|\xi\|_{\infty}$ [see hypothesis $H(\xi)$ ] and consider the Carathéodory function $\widehat{k}: \Omega \times \mathbb{R} \rightarrow \mathbb{R}$ defined by

$$
\widehat{k}(z, x)= \begin{cases}0 & \text { if } x<0 \\ f(z, x)+9 x^{p-1} & \text { if } 0 \leq x \leq w_{+}(z), \\ f\left(z, w_{+}(z)\right)+9 w_{+}(z)^{p-1} & \text { if } w_{+}(z)<x\end{cases}
$$

Let $\widehat{K}(z, x)=\int_{0}^{x} \widehat{k}(z, s) d s$ and consider the $C^{1}$-functional $\widehat{\psi}: W^{1, p}(\Omega) \rightarrow \mathbb{R}$ defined by

$$
\widehat{\psi}(u)=\frac{1}{p} \mu(u)+\frac{\vartheta}{p}\|u\|_{p}^{p}-\int_{\Omega} \widehat{K}(z, u) d z \quad \text { for all } u \in W^{1, p}(\Omega) .
$$

From (43) and since $\vartheta>\|\xi\|_{\infty}$, we see that $\widehat{\psi}$ is coercive. Also, via the Sobolev embedding theorem and the compactness of the trace map, we check that $\widehat{\psi}$ is sequentially weakly lower semicontinuous. So, by the Weierstrass-Tonelli theorem, we can find $u_{0} \in W^{1, p}(\Omega)$ such that

$$
\psi\left(u_{0}\right)=\inf \left[\psi(u): u \in W^{1, p}(\Omega)\right]
$$

Hypothesis $H(a)_{2}$ (iv) implies that we can find $c_{15} \geq 1$ and $\delta \in\left(0, c_{+}\right)$such that

$$
G(y) \leq c_{15}|y|^{q} \quad \text { for all }|y| \leq \delta
$$

Moreover, hypothesis $H_{2}$ (iv) implies that given any $\tau>0$, we can find $\delta_{1} \in(0, \delta]$ such that

$$
F(z, x) \geq \tau x^{q} \quad \text { for a.a. } z \in \Omega \text {, all } x \in\left[0, \delta_{1}\right] .
$$

Let $\widehat{u}_{1}(q)\left(\xi^{+}, \beta\right)$ be the principal eigenvalue for the eigenvalue problem (4), when the differential operator is $u \rightarrow-\Delta_{q} u+\xi^{+}(z) u$. We know that $\widehat{u}_{1}(q)\left(\xi^{+}, \beta\right) \in D_{+}$and so we can find $t \in(0,1)$ small such that

$$
t \widehat{u}_{1}(q)\left(\xi^{+}, \beta\right)(z) \in\left(0, \delta_{1}\right] \text { and } t\left|\nabla \widehat{u}_{1}(q)\left(\xi^{+}, \beta\right)(z)\right| \leq \delta_{1}
$$

for all $z \in \bar{\Omega}$.

Then, we have

$$
\begin{aligned}
& \psi\left(t \widehat{u}_{1}(q)\left(\xi^{+}, \beta\right)\right) \\
& \leq c_{15} t^{q}\left[\left\|\nabla \widehat{u}_{1}(q)\left(\xi^{+}, \beta\right)\right\|_{q}^{q}+\int_{\Omega} \xi^{+}(z) \widehat{u}_{1}(q)\left(\xi^{+}, \beta\right)^{q} d z\right. \\
& \left.\quad+\int_{\partial \Omega} \beta(z) \widehat{u}_{1}(q)\left(\xi^{+}, \beta\right)^{q} d \sigma\right]-\int_{\Omega} F\left(z, t \widehat{u}_{1}(q)\left(\xi^{+}, \beta\right)\right) d z \\
& \quad\left[\operatorname{see}(45),(47) \text { and recall } c_{14} \geq 1\right] \\
& =t^{q}\left[c_{15} \widehat{\lambda}_{1}(q)\left(\xi^{+}, \beta\right)-\tau\right] .
\end{aligned}
$$

Choosing $\tau>c_{15} \widehat{\lambda}_{1}(q)\left(\xi^{+}, \beta\right)$, we see that 


$$
\begin{aligned}
& \psi\left(\widehat{u}_{1}(q)\left(\xi^{+}, \beta\right)\right)<0 \\
\Rightarrow \quad & \psi\left(u_{0}\right)<0=\psi(0) \quad[\operatorname{see}(44)] \\
\Rightarrow \quad & u_{0} \neq 0 .
\end{aligned}
$$

From (44), we have

$$
\begin{aligned}
& \psi^{\prime}\left(u_{0}\right)=0 \\
\Rightarrow \quad & \left\langle A\left(u_{0}\right), h\right\rangle+\int_{\Omega}(\xi(z)+\vartheta)\left|u_{0}\right|^{p-2} u_{0} h d z \\
& +\int_{\partial \Omega} \beta(z)\left|u_{0}\right|^{p-2} u_{0} h d \sigma=\int_{\Omega} \widehat{k}\left(z, u_{0}\right) h d z
\end{aligned}
$$

for all $h \in W^{1, p}(\Omega)$. In (48), we choose $h=-u_{0}^{-} \in W^{1, p}(\Omega)$. Then,

$$
\begin{aligned}
& \frac{c_{1}}{p-1}\left\|\nabla u_{0}^{-}\right\|_{p}^{p}+\int_{\Omega}(\xi(z)+\vartheta)\left(u_{0}^{-}\right)^{p} d z \leq 0 \\
& {[\text { see Lemma } 1 \text {, hypothesis } H(\beta) \text { and }(43)] } \\
\Rightarrow \quad & \left.c_{16}\left\|u_{0}^{-}\right\|^{p} \leq 0 \text { for some } c_{16}>0 \text { (recall that } \vartheta>\|\xi\|_{\infty}\right) \\
\Rightarrow & u_{0} \geq 0, u_{0} \neq 0 .
\end{aligned}
$$

Next, in (48), we choose $h=\left(u_{0}-w_{+}\right)^{+} \in W^{1, p}(\Omega)$. Then,

$$
\begin{aligned}
& \left\langle A\left(u_{0}\right),\left(u_{0}-w_{+}\right)^{+}\right\rangle+\int_{\Omega}(\xi(z)+\vartheta) u_{0}^{p-1}\left(u_{0}-w_{+}\right)^{+} d z \\
& +\int_{\partial \Omega} \beta(z) u_{0}^{p-1}\left(u_{0}-w_{+}\right)^{+} d \sigma \\
& =\int_{\Omega} \widehat{k}\left(z, u_{0}\right)\left(u_{0}-w_{+}\right)^{+} d z \\
& =\int_{\Omega}\left[f\left(z, w_{+}\right)+\vartheta w_{+}^{p-1}\right]\left(u_{0}-w_{+}\right)^{+} d z \quad[\operatorname{see}(43)] \\
& \leq\left\langle A\left(w_{+}\right),\left(u_{0}-w_{+}\right)^{+}\right\rangle+\int_{\Omega}(\xi(z)+\vartheta) w_{+}^{p-1}\left(u_{0}-w_{+}\right)^{+} d z \\
& \quad+\int_{\partial \Omega} \beta(z) w_{+}^{p-1}\left(u_{0}-w_{+}\right)^{+} d \sigma \\
& \quad\left[\text { see hypotheses } H_{2}(v) \text { and } H(\beta)\right] \\
& \Rightarrow \quad u_{0} \leq w_{+} \quad\left(\text { since } \vartheta>\|\xi\|_{\infty}\right) .
\end{aligned}
$$

So, we have proved that

$$
u_{0} \in\left[0, w_{+}\right], \quad u_{0} \neq 0 .
$$

From (49) and (43), Eq. (48) becomes (see Ref. 30)

$$
\begin{aligned}
& \left\langle A\left(u_{0}\right), h\right\rangle+\int_{\Omega} \xi(z) u_{0}^{p-1} h d z+\int_{\partial \Omega} \beta(z) u_{0}^{p-1} h d \sigma \\
& =\int_{\Omega} f\left(z, u_{0}\right) h d z \\
& \text { for all } h \in W^{1, p}(\Omega) \\
\Rightarrow & \begin{cases}-\operatorname{div} a\left(\nabla u_{0}(z)\right)+\xi(z) u_{0}(z)^{p-1}=f\left(z, u_{0}(z)\right) \\
\frac{\partial u_{0}}{\partial n_{a}}+\beta(z) u_{0}^{p-1}=0 \text { on } \partial \Omega & \text { for a.a. } z \in \Omega,\end{cases}
\end{aligned}
$$

As before from (50) and the nonlinear regularity theory (see Refs. 18, 22, and 32), we have $u_{0} \in C_{+} \backslash\{0\}$. On account of hypothesis $H_{2}$ (vi), there is $\tilde{\xi}_{+}>\max \left\{\widehat{\xi}_{+},\|\xi\|_{\infty}\right\}$ such that $f(z, x)+\tilde{\xi}_{+} x^{p-1} \geq 0$ for a.a. $z \in \Omega$, all $x \in\left[0,\left\|w_{+}\right\|_{\infty}\right]$. From (50), we have the following equation (see Ref. 30, pp. 111 and 120): 


$$
\begin{aligned}
& \operatorname{div} a\left(\nabla u_{0}(z)\right) \leq\left[\|\xi\|_{\infty}+\tilde{\xi}_{+}\right] u_{0}(z)^{p-1} \quad \text { for a.a. } z \in \Omega \\
\Rightarrow & u_{0} \in D_{+} .
\end{aligned}
$$

In addition, note that

$$
\begin{aligned}
& -\operatorname{div} a\left(\nabla u_{0}(z)\right)+\left(\xi(z)+\tilde{\xi}_{+}\right) u_{0}(z)^{p-1} \\
& =f\left(z, u_{0}(z)\right)+\tilde{\xi}_{+} u_{0}(z)^{p-1} \\
& \leq f\left(z, w_{+}(z)\right)+\tilde{\xi}_{+} w_{+}(z)^{p-1} \\
& {\left[\text { see hypothesis } H_{2}\left(\text { vi) and recall that } u_{0} \leq w_{+}\right]\right.} \\
& \leq-c_{14}+\left(\xi(z)+\tilde{\xi}_{+}\right) w_{+}(z)^{p-1} \\
& \leq-\operatorname{div} a\left(\nabla w_{+}(z)\right)+\left(\xi(z)+\tilde{\xi}_{+}\right) w_{+}(z)^{p-1} \text { for a.a. } z \in \Omega \\
& {\left[\text { see hypothesis } H_{2}(\text { iv })\right] .}
\end{aligned}
$$

From (51) and Proposition 5 of Ref. 10, we have

$$
\begin{aligned}
& \left(w_{+}-u_{0}\right)(z)>0 \text { for all } z \in \Omega, \\
& \left.\frac{\partial\left(w_{+}-u_{0}\right)}{\partial n}\right|_{\Sigma_{0}}<0 \text { with } \Sigma_{0}=\left\{z \in \partial \Omega: u_{0}(z)=w_{+}(z)\right\} \\
\Rightarrow & w_{+}-u_{0} \in \widehat{D}_{+}\left(\Sigma_{0}\right) .
\end{aligned}
$$

Using $u_{0}$, we introduce the following Carathéodory function:

$$
\widehat{\gamma}_{0}(z, x)= \begin{cases}f\left(z, u_{0}(z)\right)+\vartheta u_{0}(z)^{p-1} & \text { if } x \leq u_{0}(z), \\ f(z, x)+\vartheta x^{p-1} & \text { if } u_{0}(z)<x\end{cases}
$$

We set $\widehat{\Gamma}_{0}(z, x)=\int_{0}^{x} \gamma_{0}(z, s) d s$ and consider the $C^{1}$-functional $\widehat{\tau}_{0}: W^{1, p}(\Omega) \rightarrow \mathbb{R}$ defined by

$$
\widehat{\tau}_{0}(u)=\frac{1}{p} \mu(u)+\frac{\vartheta}{p}\|u\|_{p}^{p}-\int_{\Omega} \widehat{\Gamma}_{0}(z, u) d z \quad \text { for all } u \in W^{1, p}(\Omega) .
$$

Using (53), we can show that

$$
K_{\widehat{\tau}_{0}} \subseteq\left[u_{0}\right) \cap D_{+}=\left\{u \in C^{1}(\bar{\Omega}): u_{0}(z) \leq u(z) \text { for all } z \in \bar{\Omega}\right\} .
$$

We may assume that

$$
K_{\widehat{\tau}_{0}} \cap\left[u_{0}, w_{+}\right]=\left\{u_{0}\right\} .
$$

Indeed, if we can find $\tilde{u}_{0} \in K_{\widehat{\tau}_{0}} \cap\left[u_{0}, w_{+}\right], \tilde{u}_{0} \neq u_{0}$, then from (54) we have

$$
\begin{aligned}
& u_{0} \leq \tilde{u}_{0}, \tilde{u}_{0} \in D_{+} \\
\Rightarrow \quad & \tilde{u}_{0} \text { is the desired second positive solution of (1) [see (53)]. }
\end{aligned}
$$

We consider the Carathédory function $\tilde{\gamma}_{0}: \Omega \times \mathbb{R} \rightarrow \mathbb{R}$ defined by

$$
\tilde{\gamma}_{0}(z, x)= \begin{cases}\widehat{\gamma}_{0}(z, x) & \text { if } x \leq w_{+}(z) \\ \widehat{\gamma}_{0}\left(z, w_{+}(z)\right) & \text { if } w_{+}(z)<x .\end{cases}
$$

We set $\tilde{\Gamma}_{0}(z, x)=\int_{0}^{x} \tilde{\gamma}_{0}(z, s) d s$ and consider the $C^{1}$-functional $\tilde{\tau}_{0}: W^{1, p}(\Omega) \rightarrow \mathbb{R}$ defined by

$$
\tilde{\tau}_{0}(u)=\frac{1}{p} \mu(u)+\frac{\vartheta}{p}\|u\|_{p}^{p}-\int_{\Omega} \tilde{\Gamma}_{0}(z, u) d z \quad \text { for all } u \in W^{1, p}(\Omega) .
$$

As before, since $9>\|\xi\| \infty$ and using (53) and (56), we have that $\widehat{\tau}_{0}(\cdot)$ is coercive and sequentially weakly lower semicontinuous. So, via the Weierstrass-Tonelli theorem, we can find $\tilde{u}_{0} \in W^{1, p}(\Omega)$ such that 


$$
\tilde{\tau}_{0}\left(\tilde{u}_{0}\right)=\inf \left[\tilde{\tau}_{0}(u): u \in W^{1, p}(\Omega)\right]
$$

Using (53) and (56), we see that

$$
\begin{aligned}
& K_{\widehat{\tau}_{0}} \subseteq\left[u_{0}, w_{+}\right] \cap D_{+} \\
\Rightarrow \quad & \tilde{u}_{0} \in\left[u_{0}, w_{+}\right] \cap D_{+} \quad[\operatorname{see}(57)] .
\end{aligned}
$$

Note that

$$
\left.\widehat{\tau}_{0}^{\prime}\right|_{\left[0, w_{+}\right]}=\left.\tilde{\tau}_{0}^{\prime}\right|_{\left[0, w_{+}\right]} \quad[\text { see (53) and (56)]. }
$$

From (55), (58), and (59), it follows that $\tilde{u}=u_{0}$. Then, (52) and Proposition 1 imply that

$$
u_{0} \text { is a local } W^{1, p}(\Omega)-\text { minimizer of } \widehat{\tau}_{0} .
$$

We assume that $K_{\widehat{\tau}_{0}}$ is finite [otherwise, on account of (54), we have an infinity of positive smooth solutions of (1) bigger than $u_{0}$ and so we are done]. Then, we can find $\rho \in(0,1)$ small such that

$$
\widehat{\tau}_{0}\left(u_{0}\right)<\inf \left[\widehat{\tau}_{0}(u):\left\|u-u_{0}\right\|=\rho\right]=\widehat{m}_{\rho}
$$

(see Ref. 1, Proof of Proposition 29). Hypothesis $\mathrm{H}_{2}$ (ii) implies that for all $u \in D_{+}$, we have

$$
\widehat{\tau}_{0}(t u) \rightarrow-\infty \quad \text { as } \quad t \rightarrow+\infty .
$$

Note that

$$
\begin{aligned}
& \left.\widehat{\tau}_{0}\right|_{\left[u_{0}\right)}=\left.\widehat{\varphi}\right|_{\left[u_{0}\right)}+\widehat{\xi}_{*} \text { for some } \widehat{\xi}_{*} \in \mathbb{R}[\operatorname{see}(53) \text { and (8) }] \\
\Rightarrow & \left.\widehat{\tau}_{0} \text { satisfies the } C-\text { condition (see Proposition } 5\right) .
\end{aligned}
$$

Then (61), (62), and (63) permit the use of Theorem 1 (the mountain pass theorem). So, we can find $\widehat{u} \in W^{1, p}(\Omega)$ such that

$$
\widehat{u} \in K_{\widehat{\tau}_{0}} \subseteq\left[u_{0}\right) \cap D_{+} \quad[\operatorname{see}(54)], \quad \widehat{m}_{\rho} \leq \widehat{\tau}_{0}(\widehat{u}) .
$$

From (61) and (64), it follows that $\widehat{u} \neq u_{0}, u_{0} \leq \widehat{u}$, and $\widehat{u}$ is a positive smooth solution of (1).

In the current setting, we can prove the existence of a smallest positive solution for problem (1). Let $S_{+}$be the set of positive solutions for problem (1). We have seen that under hypotheses $H(a), H(\beta)$, and $H_{2}$, we have $\emptyset \neq S_{+} \subseteq D_{+}$. Moreover, from Ref. 33 (see also Ref. 9), we have that $S_{+}$is downward directed (that is, if $u_{1}, u_{2} \in S_{+}$, then we can find $u \in S_{+}$such that $u \leq u_{1}, u \leq u_{2}$ ).

Theorem 4. If hypotheses $H(a)_{2}, H(\xi), H(\beta)$, and $H_{2}$ hold, then problem (1) admits a smallest positive solution $u_{*} \in D_{+}$.

Proof. From Lemma 3.10, p. 178 of Ref. 17, we know that we can find $\left\{u_{n}\right\}_{n \in \mathbb{N}} \subseteq S_{+}$such that

$$
\inf S_{+}=\inf _{n \in \mathbb{N}} u_{n}
$$

Evidently, we may assume that $u_{n} \in\left[0, w_{+}\right]$for all $n \in \mathbb{N}$. So, it follows that $\left\{u_{n}\right\}_{n \in \mathbb{N}} \subseteq W^{1, p}(\Omega)$ is bounded. So, by passing to a suitable subsequence if necessary, we may assume that

$$
u_{n} \stackrel{w}{\longrightarrow} u_{*} \text { in } W^{1, p}(\Omega) \text { and } u_{n} \rightarrow u_{*} \text { in } L^{r}(\Omega) \text { and in } L^{p}(\partial \Omega) .
$$

We have

$$
\begin{aligned}
\left\langle A\left(u_{n}\right), h\right\rangle+\int_{\Omega} \xi(z) u_{n}^{p-1} h d z+ & \int_{\partial \Omega} \beta(z) u_{n}^{p-1} h d \sigma \\
& =\int_{\Omega} f\left(z, u_{n}\right) h d z
\end{aligned}
$$

for all $h \in W^{1, p}(\Omega)$, all $n \in \mathbb{N}$.

In (66), we choose $h=u_{n}-u_{*} \in W^{1, p}(\Omega)$, pass to the limit as $n \rightarrow+\infty$, and use (65). Then,

$$
\lim _{n \rightarrow+\infty}\left\langle A\left(u_{n}\right), u_{n}-u_{*}\right\rangle=0
$$




$$
\Rightarrow u_{n} \rightarrow u_{*} \text { in } W^{1, p}(\Omega) \quad(\text { see Proposition } 2) .
$$

So, if in (66) we pass to the limit as $n \rightarrow+\infty$ and we use (67), then

$$
\begin{aligned}
& \left\langle A\left(u_{*}\right), h\right\rangle+\int_{\Omega} \xi(z) u_{*}^{p-1} h d z+\int_{\partial \Omega} \beta(z) u_{*}^{p-1} h d \sigma \\
= & \int_{\Omega} f\left(z, u_{*}\right) h d z \text { for all } h \in W^{1, p}(\Omega) \\
\Rightarrow \quad & u_{*} \in S_{+} \cup\{0\} .
\end{aligned}
$$

We need to show that $u_{*} \neq 0$. Hypotheses $H_{2}$ (i) and (iv) imply that given any $\lambda>0$, we can find $c_{17}=c_{17}(\lambda)>0$ such that

$$
f(z, x) \geq \lambda x^{q-1}-c_{17} x^{r-1} \quad \text { for a.a. } z \in \Omega \text {, all } \mathrm{x} \geq 0 .
$$

This unilateral growth estimate on the reaction term $f(z, \cdot)$ leads to the following auxiliary nonlinear Robin problem. Here, as before $\vartheta>\|\xi\| \infty$,

$$
\left\{\begin{array}{c}
-\operatorname{div} a(\nabla u(z))+\xi^{+}(z) u(z)^{p-1}=\lambda u(z)^{q-1}-c_{17} u(z)^{r-1} \\
\frac{\partial u}{\partial n_{a}}+\beta(z) u^{p-1}=0 \text { on } \partial \Omega, u \geq 0 .
\end{array}\right.
$$

Claim 1. For $\lambda>0$ big, problem (69) admits a unique positive solution $\bar{u} \in D_{+}$.

First, we show the existence of a positive solution for problem (69). To this end, let $J: W^{1, p}(\Omega) \rightarrow \mathbb{R}$ be the $C^{1}$-functional defined by

$$
J(u)=\frac{1}{p} \mu(u)+\frac{\vartheta}{p}\left\|u^{-}\right\|_{p}^{p}+\frac{c_{17}}{r}\left\|u^{+}\right\|_{r}^{r}-\frac{\lambda}{q}\left\|u^{+}\right\|_{q}^{q} .
$$

Recall that $\vartheta>\|\xi\|_{\infty}$. Therefore, since $q \leq p<r$, it follows that $J(\cdot)$ is coercive. In addition, the Sobolev embedding theorem and the compactness of the trace map imply that $J(\cdot)$ is sequentially weakly lower semicontinuous. So, using the Weierstrass-Tonelli theorem, we can find $\bar{u} \in W^{1, p}(\Omega)$ such that

$$
J(\bar{u})=\inf \left[J(u): u \in W^{1, p}(\Omega)\right] .
$$

Hypothesis $H(a)_{2}(i v)$ and Corollary 1 imply that there exists $c_{18}>0$ such that

$$
G(y) \leq c_{18}\left(|y|^{q}+|y|^{p}\right) \quad \text { for all } y \in \mathbb{R}^{N} .
$$

Then, for $u \in W^{1, p}(\Omega)$ with $u \geq 0,0<\|u\| \leq 1$, we have

$$
\begin{aligned}
J(u) & \leq c_{18}\left(\|\nabla u\|_{q}^{q}+\|\nabla u\|_{p}^{p}\right)+\frac{1}{p} \int_{\Omega} \xi^{+}(z) u^{p} d z \\
& +\frac{1}{p} \int_{\partial \Omega} \beta(z) u^{p} d \sigma+c_{19}\|u\|^{r}-\frac{\lambda}{q}\|u\|^{q} \text { for some } c_{19}>0 \\
& \leq\left[c_{20}-\frac{\lambda}{q}\right]\|u\|^{q} \quad(\text { recall } q \leq p<r \text { and }\|u\|=1) .
\end{aligned}
$$

However, $\lambda>0$ is arbitrary. So, choosing $\lambda>q c_{20}>0$, we have

$$
\begin{aligned}
& J(u)<0=J(0) \\
\Rightarrow & J(\bar{u})<0=J(0) \quad[\operatorname{see}(70)] \\
\Rightarrow & \bar{u} \neq 0 .
\end{aligned}
$$

From (70), we have

$$
\begin{aligned}
& J^{\prime}(\bar{u})=0 \\
& \Rightarrow \quad\langle A(\bar{u}), h\rangle+\int_{\Omega} \xi^{+}(z)|\bar{u}|^{p-2} \bar{u} h d z+\int_{\partial \Omega} \beta(z)|\bar{u}|^{p-2} \bar{u} h d \sigma \\
&=\lambda \int_{\Omega}\left(\bar{u}^{+}\right)^{p-1} h d z-c_{17} \int_{\Omega}\left(\bar{u}^{+}\right)^{r-1} h d z \\
& \quad \text { for all } h \in W^{1, p}(\Omega) .
\end{aligned}
$$


In (72), we choose $h=-\bar{u}^{-} \in W^{1, p}(\Omega)$. Then,

$$
\begin{aligned}
& \frac{c_{1}}{p-1}\left\|\nabla \bar{u}^{-}\right\|_{p}^{p}+\int_{\Omega} \xi^{+}(z)\left(\bar{u}^{-}\right)^{p} d z+\int_{\partial \Omega} \beta(z)\left(\bar{u}^{-}\right)^{p} d \sigma \leq 0 \\
& (\text { see Lemma 1) } \\
\Rightarrow & \left.c_{21}\left\|\bar{u}^{-}\right\|^{p} \leq 0 \text { for some } c_{21}>0 \text { (recall that } \vartheta>\|\xi\|_{\infty}\right) \\
\Rightarrow & \bar{u} \geq 0, \bar{u} \neq 0 .
\end{aligned}
$$

Therefore, (72) becomes (see Ref. 30)

$$
\begin{aligned}
& \langle A(\bar{u}), h\rangle+\int_{\Omega} \xi^{+}(z) \bar{u}^{p-1} h d z+\int_{\partial \Omega} \beta(z) \bar{u}^{p-1} h d \sigma \\
= & \lambda \int_{\Omega} \bar{u}^{q-1} h d z-c_{17} \int_{\Omega} \bar{u}^{r-1} h d z \text { for all } h \in W^{1, p}(\Omega) \\
\Rightarrow & \left\{\begin{array}{l}
-\operatorname{div} a(\nabla \bar{u}(z))+\xi^{+}(z) \bar{u}(z)^{p-1}=\lambda \bar{u}(z)^{q-1}-c_{17} \bar{u}(z)^{r-1} \\
\frac{\partial \bar{u}}{\partial n_{a}}+\beta(z) \bar{u}^{p-1}=0 \text { on } \partial \Omega,
\end{array}\right. \\
\Rightarrow & \quad \text { for a.a. } z \in \Omega,
\end{aligned}
$$

From (73), we have the following equation (see Ref. 36, p. 111 and 120):

$$
\begin{aligned}
\quad & \operatorname{div} a(\nabla \bar{u}(z)) \leq\left[\|\xi\|_{\infty}+c_{17}\|\bar{u}\|_{\infty}^{r-p}\right] \bar{u}(z)^{p-1} \text { for a.a. } z \in \Omega \\
\Rightarrow \quad & \bar{u} \in D_{+} .
\end{aligned}
$$

This proves the existence of a positive solution in $D_{+}$for the auxiliary problem (69), when $\lambda>0$ is big. Next, we show the uniqueness of this positive solution. To this end, we introduce the integral $j: L^{1}(\Omega) \rightarrow \overline{\mathbb{R}}=\mathbb{R} \cup\{+\infty\}$, defined by

$$
j(u)=\left\{\begin{array}{c}
\int_{\Omega} G\left(\nabla u^{1 / q}\right) d z+\frac{1}{p} \int_{\Omega} \xi^{+}(z) u^{p / q} d z \\
+\frac{1}{p} \int_{\partial \Omega} \beta(z) u^{p / q} d \sigma \quad \text { if } u^{1 / q} \in W^{1, p}(\Omega), u \geq 0, \\
+\infty \text { otherwise. }
\end{array}\right.
$$

Let $u_{1}, u_{2} \in \operatorname{dom} j=\left\{u \in L^{1}(\Omega): j(u)<+\infty\right\}$ [the effective domain of $\left.j(\cdot)\right]$. We set

$$
u=\left[t u_{1}+(1-t) u_{2}\right]^{1 / q} \text { for } t \in[0,1] .
$$

Using Lemma 1 of Ref. 7, we have

$$
\begin{array}{cc} 
& \begin{array}{l}
|\nabla u(z)| \leq\left[t\left|\nabla u_{1}(z)^{1 / q}\right|^{q}+(1-t)\left|\nabla u_{2}(z)^{1 / q}\right|^{q}\right]^{1 / q} \\
\text { for a.a. } z \in \Omega
\end{array} \\
\Rightarrow \quad & G_{0}(|\nabla u(z)|) \\
& \leq G_{0}\left(\left[t\left|\nabla u_{1}(z)^{1 / q}\right|^{q}+(1-t)\left|\nabla u_{2}(z)^{1 / q}\right|^{q}\right]^{1 / q}\right) \\
& \text { for a.a. } z \in \Omega\left[\text { recall that } G_{0}(\cdot) \text { is increasing }\right] \\
& \leq t G_{0}\left(\left|\nabla u_{1}(z)^{1 / q}\right|\right)+(1-t) G_{0}\left(\left|\nabla u_{2}(z)^{1 / q}\right|\right) \\
& \quad \text { for a.a. } z \in \Omega \\
\Rightarrow \quad & G(\nabla u(z)) \leq t G\left(\nabla u_{1}(z)^{1 / q}\right)+(1-t) G\left(\nabla u_{2}(z)^{1 / q}\right) \\
\Rightarrow \quad & j(\cdot) \text { is convex } \quad \text { for a.a. } z \in \Omega, \\
& {[\text { recall that } q \leq p \text { and see hypothesis } H(\beta)] .}
\end{array}
$$


Also by Fatou's lemma, $j(\cdot)$ is lower semicontinuous. Suppose that $\bar{v} \in W^{1, p}(\Omega)$ is another positive solution of the auxiliary problem (69). Again we show that $\bar{v} \in D_{+}$. Then, for $h=\bar{u}^{q}-\bar{v}^{q}$ and $t \in(0,1]$, small, we have $\bar{u}^{q}-t h, \bar{v}^{q}+t h \in \operatorname{dom} j$. Evidently, $j(\cdot)$ is Gateaux differentiable at $\bar{u}^{q}$ and at $\bar{v}^{q}$ in the direction $h$. So, from the chain rule and the nonlinear Green's identity (see Ref. 12, p. 210), we obtain

$$
\begin{aligned}
& j^{\prime}\left(\bar{u}^{q}\right)(h)=\frac{1}{q} \int_{\Omega} \frac{-\operatorname{div} a(\nabla \bar{u})+\xi^{+}(z) \bar{u}^{p-1}}{\bar{u}^{q-1}} h d z, \\
& j^{\prime}\left(\bar{v}^{q}\right)(h)=\frac{1}{q} \int_{\Omega} \frac{-\operatorname{div} a(\nabla \bar{v})+\xi^{+}(z) \bar{v}^{p-1}}{\bar{v}^{q-1}} h d z,
\end{aligned}
$$

for this $h \in C^{1}(\bar{\Omega})$. From the convexity of $j(\cdot)$, we have the monotonicity of $j^{\prime}(\cdot)$. Hence,

$$
\begin{aligned}
0 & \leq \int_{\Omega}\left(\frac{-\operatorname{div} a(\nabla \bar{u})}{\bar{u}^{q-1}}-\frac{-\operatorname{div} a(\nabla \bar{v})}{\bar{v}^{q-1}}\right)\left(\bar{u}^{q}-\bar{v}^{q}\right) d z \\
& =-\int_{\Omega} \xi^{+}(z)\left(\bar{u}^{p-q}-\bar{v}^{p-q}\right)\left(\bar{u}^{q}-\bar{v}^{q}\right) d z \\
& -\int_{\Omega} c_{17}\left(\bar{u}^{r-q}-\bar{v}^{r-q}\right)\left(\bar{u}^{q}-\bar{v}^{q}\right) d z \\
\Rightarrow \quad \bar{u} & =\bar{v} \quad(\text { since } q \leq p<r) .
\end{aligned}
$$

This proves Claim 1.

Claim 2. $\bar{u} \leq u$ for all $u \in S_{+}$.

Let $u \in S_{+}$and consider the Carathédory function $k_{+}: \Omega \times \mathbb{R} \rightarrow \mathbb{R}$ defined by

$$
k_{+}(z, x)= \begin{cases}0 & \text { if } x<0, \\ \lambda x^{q-1}-c_{17} x^{r-1}+9 x^{p-1} & \text { if } 0 \leq x \leq u(z), \\ \lambda u(z)^{q-1}-c_{17} u(z)^{r-1}+\vartheta u(z)^{p-1} & \text { if } u(z)<x .\end{cases}
$$

We set $K_{+}(z, x)=\int_{0}^{x} k_{+}(z, s) d s$ and consider the $C^{1}$-functional $\psi_{+}: W^{1, p}(\Omega) \rightarrow \mathbb{R}$ defined by

$$
\begin{aligned}
& \psi_{+}(u)=\int_{\Omega} G(\nabla u) d z+\frac{1}{p} \int_{\Omega}\left(\xi^{+}(z)+\vartheta\right)|u|^{p} d z \\
& +\frac{1}{p} \int_{\partial \Omega} \beta(z)|u|^{p} d \sigma-\int_{\Omega} K_{+}(z, u) d z
\end{aligned}
$$

for all $u \in W^{1, p}(\Omega)$. Since $\vartheta>\|\xi\|_{\infty}$, from (74), we infer that $\psi_{+}$is coercive. In addition, it is sequentially weakly lower semicontinuous. So, we can find $\tilde{u} \in W^{1, p}(\Omega)$ such that

$$
\psi_{+}(\tilde{u})=\inf \left[\psi_{+}(u): u \in W^{1, p}(\Omega)\right]
$$

As in the prove of Claim 1, we show that

$$
\begin{aligned}
& \psi_{+}(\tilde{u}) \leq 0=\psi_{+}(0) \\
\Rightarrow \quad & \tilde{u} \neq 0 .
\end{aligned}
$$

From (75), we have

$$
\begin{aligned}
& \psi_{+}^{\prime}(\tilde{u})=0 \\
& \Rightarrow \quad\langle A(\tilde{u}), h\rangle+\int_{\Omega}\left(\xi^{+}(z)+\vartheta\right)|\tilde{u}|^{p-2} \tilde{u} h d z \\
&+\int_{\partial \Omega} \beta(z)|\tilde{u}|^{p-2} \tilde{u} h d \sigma=\int_{\Omega} k_{+}(z, \tilde{u}) h d z
\end{aligned}
$$

for all $h \in W^{1, p}(\Omega)$. In (76), first we choose $h=-\tilde{u}^{-} \in W^{1, p}(\Omega)$. Then, since $\vartheta>\|\xi\|_{\infty}$, we obtain

$$
\begin{aligned}
& c_{22}\left\|\tilde{u}^{-}\right\| \leq 0 \quad \text { for some } c_{22}>0 \\
\Rightarrow \quad \tilde{u} \geq 0, \tilde{u} \neq 0 . &
\end{aligned}
$$


Also in (76), we choose $h=(\tilde{u}-u)^{+} \in W^{1, p}(\Omega)$. Then,

$$
\begin{aligned}
&\left\langle A(\tilde{u}),(\tilde{u}-u)^{+}\right\rangle+\int_{\Omega}\left(\xi^{+}(z)+\vartheta\right) \tilde{u}^{p-1}(\tilde{u}-u)^{+} d z \\
&+\int_{\partial \Omega} \beta(z) \tilde{u}^{p-1}(\tilde{u}-u)^{+} d \sigma \\
&= \int_{\Omega} k_{+}(z, \tilde{u})(\tilde{u}-u)^{+} d z \\
&= \int_{\Omega}\left[\lambda u^{q-1}-c_{17} u^{r-1}+\vartheta u^{p-1}\right](\tilde{u}-u)^{+} d z \\
& \leq \int_{\Omega}\left[f(z, u)+\vartheta u^{p-1}\right](\tilde{u}-u)^{+} d z \quad[\operatorname{see}(68)] \\
& \leq\left\langle A(u),(\tilde{u}-u)^{+}\right\rangle+\int_{\Omega}\left(\xi^{+}(z)+\vartheta\right) u^{p-1}(\tilde{u}-u)^{+} d z \\
&+\int_{\partial \Omega} \beta(z) u^{p-1}(\tilde{u}-u)^{+} d \sigma\left(\text { since } u \in S_{+}\right) \\
& \Rightarrow \quad\left\langle A(\tilde{u})-A(u),(\tilde{u}-u)^{+}\right\rangle \\
&+\int_{\Omega}\left(\xi^{+}(z)+\vartheta\right)\left(\tilde{u}^{p-1}-u^{p-1}\right)(\tilde{u}-u)^{+} d z \\
&+\int_{\partial \Omega} \beta(z)\left(\tilde{u}^{p-1}-u^{p-1}\right)(\tilde{u}-u)^{+} d \sigma \leq 0 \\
& \Rightarrow \quad \tilde{u} \leq u\left[\operatorname{since} \vartheta \geq\|\xi\|_{\infty}, \text { see also hypothesis } H(\beta)\right] .
\end{aligned}
$$

So, we have proved that

$$
\tilde{u} \in[0, u], \tilde{u} \neq 0 .
$$

Then, on account of (74), Eq. (76) becomes

$$
\begin{aligned}
& \langle A(\tilde{u}), h\rangle+\int_{\Omega} \xi^{+}(z) \tilde{u}^{p-1} h d z+\int_{\partial \Omega} \beta(z) \tilde{u}^{p-1} h d \sigma \\
= & \int_{\Omega}\left(\lambda \tilde{u}^{q-1}-c_{17} \tilde{u}^{r-1}\right) h d z \text { for all } h \in W^{1, p}(\Omega) \\
\Rightarrow \quad & \tilde{u} \text { is a positive solution of the auxiliary problem (69). }
\end{aligned}
$$

From Claim 1, we have

$$
\begin{aligned}
& \tilde{u}=\bar{u} \in D_{+} \\
\Rightarrow \quad \bar{u} & \leq u \quad \text { for all } u \in S_{+}[\operatorname{see}(77)] .
\end{aligned}
$$

This proves Claim 2. Now, on account of Claim 2, we have

$$
\begin{aligned}
& \bar{u} \leq u_{n} \quad \text { for all } n \in \mathbb{N} \\
\Rightarrow & \bar{u} \leq u_{*} \quad[\operatorname{see}(67)] \\
\Rightarrow & u_{*} \neq 0 \quad \text { and so } \quad u_{*} \in S_{+}, u_{*}=\inf S_{+} .
\end{aligned}
$$

\section{IV. p-LAPLACIAN EQUATION}

In the existence theorem of Sec. III (see Theorem 2), we assumed that the quotient $\frac{f(z, x)}{x^{p-1}}$ stayed below a multiple of the principal eigenvalue $\widehat{\lambda}_{1}(\widehat{\xi}, \widehat{\beta})$. In the particular case of the $p$-Laplacian [that is, $a(y)=|y|^{p-2} y$ for all $y \in \mathbb{R}^{N}$ ], hypothesis $H_{1}$ (iv) becomes

$$
\begin{gathered}
\tilde{\eta}(z) \leq \liminf _{x \rightarrow 0^{+}} \frac{f(z, x)}{x^{p-1}} \leq \limsup _{x \rightarrow 0^{+}} \frac{f(z, x)}{x^{p-1}} \leq \eta(z) \\
\text { uniformily for a.a. } z \in \Omega, \\
\eta(z) \leq \widehat{\lambda}_{1}(\xi, \beta) \quad \text { for a.a. } z \in \Omega, \eta \neq \widehat{\lambda}_{1}(\xi, \beta) .
\end{gathered}
$$


So, at zero, we have nonuniform, nonresonance with respect to the principal eigenvalue $\widehat{\lambda}_{1}(\xi, \beta)$. This condition made $u=0$ a local minimizer of $\widehat{\varphi}$ (see Proposition 6) and made possible the use of Theorem 1 (the mountain pass theorem). It is natural to ask what happens if the quotient $\frac{f(z, x)}{x^{p-1}}$ stays above $\widehat{\lambda}_{1}(\xi, \beta)$ as $x \rightarrow 0^{+}$. We will show that in this case for the $p$-Laplacian equation, we can still produce a nontrivial smooth solution but without any information on its sign. In this case, the mountain pass theorem cannot be used. Instead we use tools from Morse theory (critical groups). So, the problem under consideration is the following nonlinear Robin equation:

$$
\text { (1) } P \quad\left\{\begin{array}{cc}
-\Delta_{p} u(z)+\xi(z)|u(z)|^{p-2} u(z)=f(z, u(z)) & \text { in } \Omega, \\
\frac{\partial u}{\partial n_{p}}+\beta(z)|u|^{p-2} u=0 & \text { on } \partial \Omega .
\end{array}\right.
$$

Therefore, now we have

$$
\begin{aligned}
& a(y)=|y|^{p-2} y \quad \text { for all } y \in \mathbb{R}^{N}(1<p<\infty), \\
& G(y)=\frac{1}{p}|y|^{p} \quad \text { for all } y \in \mathbb{R}^{N} \\
& \frac{\partial u}{\partial n_{p}}=|u|^{p-2} \frac{\partial u}{\partial n} \quad \text { for all } u \in W^{1, p}(\Omega)
\end{aligned}
$$

We introduce the following subspace of $W^{1, p}(\Omega)$ :

$$
V=\left\{u \in W^{1, p}(\Omega): \int_{\Omega} \widehat{u}_{1}(\xi, \beta) u d z=0\right\} .
$$

We have the following direct sum decomposition:

$$
W^{1, p}(\Omega)=\mathbb{R} \widehat{u}_{1}(\xi, \beta) \oplus V
$$

In the sequel, for economy in the notation, we write $\widehat{u}_{1}=\widehat{u}_{1}(\xi, \beta) \in D_{+}$. We set

$$
\widehat{\lambda}_{V}=\inf \left[\frac{\mu_{p}(u)}{\|u\|_{p}^{p}}: u \in W^{1, p}(\Omega), u \neq 0\right] .
$$

In this case, the $C^{1}$-functional $\mu_{p}: W^{1, p}(\Omega) \rightarrow \mathbb{R}$ is given by

$$
\mu_{p}(u)=\|\nabla u\|_{p}^{p}+\int_{\Omega} \xi(z)|u|^{p} d z+\int_{\partial \Omega} \beta(z)|u|^{p} d \sigma
$$

for all $u \in W^{1, p}(\Omega)$.

As in Ref. 28 (see Proposition 3.8), we can show that

$$
\widehat{\lambda}_{1}<\widehat{\lambda}_{V} \leq \widehat{\lambda}_{2}
$$

Moreover, if $p=2$, then $\widehat{\lambda}_{V}=\widehat{\lambda}_{2}$. The hypotheses on the reaction term are the following:

$H_{3}: f: \Omega \times \mathbb{R} \rightarrow \mathbb{R}$ is a Carathédory function such that $f(z, 0)=0$ for a.a. $z \in \Omega$ and

(i) $|f(z, x)| \leq a(z)\left(1+|x|^{r-1}\right)$ for a.a. $z \in \Omega$, all $x \in \mathbb{R}$, with $a \in L^{\infty}(\Omega)_{+}, p<r<p *$;

(ii) if $F(z, x)=\int_{0}^{x} f(z, s) d s$, then $\lim _{x \rightarrow \pm \infty} \frac{F(z, x)}{|x|^{p}}=+\infty$ uniformly for a.a. $z \in \Omega$;

(iii) if $e(z, x)=f(z, x) x-p F(z, x)$, then there exists $d \in L^{1}(\Omega)$ such that $e(z, x) \leq e(z, y)+d(z)$ for a.a. $z \in \Omega$, all $0 \leq x \leq y$ or $y \leq x \leq 0$;

(iv) there exist $\delta_{0} \in(0,1)$ and $\eta \in\left(\widehat{\lambda}_{1}, \widehat{\lambda}_{V}\right)$ such that $\widehat{\lambda}_{1}|x|^{p} \leq f(z, x) x \leq \eta|x|^{p}$ for a.a. $z \in \Omega$, all $|x| \leq \delta_{0}$.

Remark 7. Now hypothesis $H_{3}(i v)$ at zero permits resonance with respect to the principal eigenvalue $\widehat{\lambda}_{1}$.

Let $\varphi_{p}: W^{1, p}(\Omega) \rightarrow \mathbb{R}$ be the energy (Euler) functional for problem $(1)_{P}$ defined by

$$
\varphi_{p}(u)=\frac{1}{p} \mu_{p}(u)-\int_{\Omega} F(z, u) d z \quad \text { for all } u \in W^{1, p}(\Omega),
$$

with $\mu_{p}: W^{1, p}(\Omega) \rightarrow \mathbb{R}$ defined by

$$
\mu_{p}(u)=\|\nabla u\|_{p}^{p}+\int_{\Omega} \xi(z)|u|^{p} d z+\int_{\partial \Omega} \beta(z)|u|^{p} d \sigma
$$


for all $u \in W^{1, p}(\Omega)$. Note that the hypotheses $H_{3}$ (i)-(iii) are the same as the corresponding hypotheses $H_{1}$ (i)-(iii). Therefore, Proposition 5 remains valid and we have the following proposition:

Proposition 9. If hypotheses $H(\xi), H(\beta)$, and $H_{3}$ hold, then the functional $\varphi$ satisfies the $C$-condition.

Next, we compute the critical groups of the energy functional at infinity. In fact, we will do this for the general case of the differential operator $\operatorname{div} a(\nabla u)$ since the result is of independent interest and can be used in other occasions. In this case, the energy functional $\varphi$ : $W^{1, p}(\Omega) \rightarrow \mathbb{R}$ is given by

$$
\varphi(u)=\frac{1}{p} \mu(u)-\int_{\Omega} F(z, u) d z \quad \text { for all } u \in W^{1, p}(\Omega)
$$

with $\mu: W^{1, p}(\Omega) \rightarrow \mathbb{R}$ defined by

$$
\mu(u)=\int_{\Omega} p G(\nabla u) d z+\int_{\Omega} \xi(z)|u|^{p} d z+\int_{\partial \Omega} \beta(z)|u|^{p} d \sigma
$$

for all $u \in W^{1, p}(\Omega)$.

As we already mentioned, if $G(y)=\frac{1}{p}|y|^{p}$ for all $y \in \mathbb{R}^{N}$, then $\varphi=\varphi_{p}$ and this energy functional corresponds to the $p$-Laplace differential operator.

Proposition 10. If hypotheses $H(a)_{1}, H(\xi), H(\beta)$, and $H_{3}$ (i)-(iii) hold, then $C_{k}(\varphi, \infty)=0$ for all $k \in \mathbb{N}_{0}$.

Proof. Let $\partial B_{1}=\left\{u \in W^{1, p}(\Omega):\|u\|=1\right\}$. Hypotheses $H_{3}(\mathrm{i})$, (ii) imply that given $k>0$ we can find $c_{23}=c_{23}(k)>0$ such that

$$
F(z, x) \geq \frac{k}{p}|x|^{p}-c_{23} \quad \text { for a.a. } z \in \Omega \text {, all } x \in \mathbb{R} \text {. }
$$

Then, for $u \in \partial B_{1}$ and $t>0$, we have

$$
\begin{aligned}
\varphi(t u) & =\frac{1}{p} \mu(t u)-\int_{\Omega} F(z, t u) d z \\
& \leq c_{5}\left(1+t^{p}\|\nabla u\|_{p}^{p}\right)+\frac{t^{p}}{p} c_{24}\|u\|_{p}^{p}-\frac{t^{p}}{p} k\|u\|_{p}^{p}+c_{23}|\Omega|_{N} \\
& \text { for some } c_{24}>0 \\
& {[\text { see Corollary } 1 \text {, hypotheses } H(\xi), H(\beta) \text { and }(82)] } \\
& \leq t^{p}\left[c_{25}-k\|u\|_{p}^{p}\right] \quad \text { for some } c_{25}>0 \quad\left(\text { recall } u \in \partial B_{1}\right) .
\end{aligned}
$$

Recall that $k>0$ is arbitrary. So, choosing $k>\frac{c_{25}}{\|u\|_{p}^{p}}$, we infer that

$$
\varphi(t u) \rightarrow-\infty \quad \text { as } t \rightarrow+\infty .
$$

For $u \in \partial B_{1}$ and $t>0$, we have

$$
\begin{aligned}
& \frac{d}{d t} \varphi(t u)=\left\langle\varphi^{\prime}(t u), u\right\rangle \quad \text { (by the chain rule) } \\
= & \frac{1}{t}\left\langle\varphi^{\prime}(t u), t u\right\rangle \\
= & \frac{1}{t}\left[\int_{\Omega}(a(t \nabla u), t \nabla u)_{\mathbb{R}^{N}} d z+\int_{\Omega} \xi(z)|t u|^{p} d z\right. \\
& \left.+\int_{\partial \Omega} \beta(z)|t u|^{p} d \sigma-\int_{\Omega} f(z, t u)(t u) d z\right] \\
\leq & \frac{1}{t}\left[\int_{\Omega} p G(t \nabla u) d z+\int_{\Omega} \xi(z)|t u|^{p} d z+\int_{\partial \Omega} \beta(z)|t u|^{p} d \sigma-\int_{\Omega} p F(z, t u) d z+c_{26}\right] \\
& \text { for some } c_{26}>0\left[\text { see hypotheses } H(a)_{1}(\text { iv) }), H_{3}(\text { iii) })\right. \\
= & \frac{1}{t}\left[p \varphi(t u)+c_{26}\right] .
\end{aligned}
$$

From (83) and (84), it follows that $\frac{d}{d t} \varphi(t u)<0$ for all $t>0$ big. The implicit function theorem implies that we can find $\widehat{e} \in C\left(\partial B_{1}\right)$ such that

$$
\widehat{e}>0 \quad \text { and } \varphi(\widehat{e}(u) u)=\rho_{0}<-\frac{c_{25}}{p} .
$$


We extend $\widehat{e}(\cdot)$ on $W^{1, p}(\Omega) \backslash\{0\}$ by $\widehat{e}_{0}(u)=\frac{1}{\|u\|} \widehat{e}\left(\frac{u}{\|u\|}\right)$ for all $u \in W^{1, p}(\Omega) \backslash\{0\}$. We have $\widehat{e}_{0} \in C\left(W^{1, p}(\Omega) \backslash\{0\}\right)$ and $\varphi\left(\widehat{e}_{0}(u) u\right)=\rho_{0}$. Also, we have

$$
\varphi(u)=\rho_{0} \Rightarrow \widehat{e}_{0}(u)=1 .
$$

So, if we define

$$
\tilde{e}_{0}(u)= \begin{cases}1 & \text { if } \varphi(u) \leq \rho_{0}, \\ \widehat{e}_{0}(u) & \text { if } \rho_{0}<\varphi(u)\end{cases}
$$

then we have $\tilde{e}_{0} \in C\left(W^{1, p}(\Omega) \backslash\{0\}\right)$ [see (86)]. We consider the deformation $h:[0,1] \times\left(W^{1, p}(\Omega) \backslash\{0\}\right) \rightarrow W^{1, p}(\Omega) \backslash\{0\}$ defined by $h(t, u)=(1-t) u+t \tilde{e}_{0}(u) u$ for all $t \in[0,1]$, all $u \in W^{1, p}(\Omega)$. We have

- $h(0, u)=u$ for all $u \in W^{1, p}(\Omega) \backslash\{0\}$,

- $h(1, u)=\tilde{e}_{0}(u) u \in \varphi^{\rho_{0}} \quad[$ see $(87)]$,

- $\left.h(t, \cdot)\right|_{\rho_{0}}=\left.i d\right|_{\varphi_{0}} \quad[$ see $(86)$ and (87)].

From these facts, we infer that

$$
\varphi^{\rho_{0}} \quad \text { is a strong deformation retract of } W^{1, p}(\Omega) \backslash\{0\} .
$$

Consider the radial retraction $r_{1}: W^{1, p}(\Omega) \backslash\{0\} \rightarrow \partial B_{1}$ defined by

$$
r_{1}(u)=\frac{u}{\|u\|} \quad \text { for all } u \in W^{1, p}(\Omega) \backslash\{0\} .
$$

We introduce the deformation $\widehat{h}:[0,1] \times\left(W^{1, p}(\Omega) \backslash\{0\}\right) \rightarrow W^{1, p}(\Omega) \backslash\{0\}$ defined by $\widehat{h}(t, u)=(1-t) u+\operatorname{tr}_{1}(u)$ for all $t \in[0,1]$, all $u \in W^{1, p}(\Omega) \backslash\{0\}$. With this deformation, we see that

$$
W^{1, p}(\Omega) \backslash\{0\} \text { is deformable into } \partial B_{1} .
$$

In addition, using radial retraction $r_{1}(\cdot)$, we see that

$$
\partial B_{1} \text { is a retract of } W^{1, p}(\Omega) \backslash\{0\} .
$$

From (89), (90), and Theorem 6.5, p. 325 of Ref. 8, we infer that

$$
\partial B_{1} \text { is a deformation retract of } W^{1, p}(\Omega) \backslash\{0\} .
$$

From (88) and (91), it follows that (see Ref. 27, p. 143)

$$
\begin{aligned}
& \varphi^{\rho_{0}} \text { and } \partial B_{1} \text { are homotopy equivalent } \\
\Rightarrow \quad & H_{k}\left(W^{1, p}(\Omega), \varphi^{\rho_{0}}\right)=H_{k}\left(W^{1, \rho}(\Omega), \partial B_{1}\right) \quad \text { for all } k \in \mathbb{N}_{0} .
\end{aligned}
$$

The Sobolov space $W^{1, p}(\Omega)$ is infinite dimensional. Hence (see Ref. 16, Problems 4.154 and 4.159, and Ref. 27, p. 147),

$$
\begin{aligned}
& \partial B_{1} \text { is contractible } \\
& (\text { see Ref. 16, Problems } 4.154 \text { and } 4.159) \\
\Rightarrow \quad & H_{k}\left(W^{1, p}(\Omega), \partial B_{1}\right)=0 \quad \text { for all } k \in \mathbb{N}_{0} \\
& (\text { see Ref. 27, p. 147) } \\
\Rightarrow \quad & H_{k}\left(W^{1, p}(\Omega), \varphi^{\rho_{0}}\right)=0 \quad \text { for all } k \in \mathbb{N}_{0} \quad[\operatorname{see}(92)] .
\end{aligned}
$$

As usual, we assume that $K_{\varphi}$ is finite (or otherwise, we already have an infinity of nontrivial solutions which are in $C^{1}(\bar{\Omega})$ by the nonlinear regularity theory). So, choosing $\rho_{0}<-\frac{c_{25}}{p}$ even more negative, we have 


$$
\begin{aligned}
C_{k}(\varphi, \infty) & =H_{k}\left(W^{1, p}(\Omega), \varphi^{\rho_{0}}\right) \quad \text { for all } k \in \mathbb{N}_{0} \\
\Rightarrow \quad C_{k}(\varphi, \infty) & =0 \quad \text { for all } k \in \mathbb{N}_{0} .
\end{aligned}
$$

Now for the functional $\varphi_{p}$, we compute the critical groups at zero.

Proposition 11. If hypotheses $H(\xi), H(\beta)$, and $H_{3}$ hold and $\widehat{\lambda}_{V}>0$, then $C_{1}\left(\varphi_{p}, 0\right) \neq 0$.

Proof. We consider the direct sum decomposition,

$$
W^{1, p}(\Omega)=\mathbb{R} \widehat{u}_{1} \oplus V \quad[\operatorname{see}(79)] .
$$

Recall that $\widehat{u}_{1} \in D_{+}$. So, for $|t| \leq 1$ small, we have

$$
\left|t \widehat{u}_{1}(z)\right| \leq \delta_{0}, \quad \text { and } \quad\left|t \nabla \widehat{u}_{1}(z)\right| \leq \delta_{0} \quad \text { for all } z \in \bar{\Omega} .
$$

Then, using (95) and hypothesis $H_{3}$ (iv), we have

$$
\varphi_{p}\left(\widehat{u}_{1}\right) \leq \frac{t^{p}}{p}\left[\mu_{p}\left(\widehat{u}_{1}\right)-\widehat{\lambda}_{1}\right]=0 \quad\left(\text { recall }\left\|\widehat{u}_{1}\right\|_{p}=1\right) .
$$

So, we can find $\rho_{1}>0$ such that

$$
\left.\varphi_{p}\right|_{\mathbb{R} \bar{u}_{1} \cap \partial \bar{B}_{\rho_{1}}} \leq 0
$$

with $\bar{B}_{\rho_{1}}=\left\{u \in W^{1, p}(\Omega):\|u\| \leq \rho_{1}\right\}$. From hypotheses $H_{3}(\mathrm{i})$, (iv), we have

$$
F(z, x) \leq \frac{\eta}{p}|x|^{p}+c_{26}|x|^{r} \text { for a.a. } z \in \Omega \text {, all } x \in \mathbb{R} \text {, some } c_{26}>0 .
$$

Then, for $u \in V$, we have

$$
\begin{aligned}
\varphi_{p}(u) & \geq \frac{1}{p}\left[\mu_{p}(u)-\eta\|u\|_{p}^{p}\right]-c_{27}\|u\|^{r} \\
& \text { for some } c_{27}>0[\operatorname{see}(97)] \\
& \geq \frac{1}{p}\left[1-\frac{\eta}{\widehat{\lambda}_{V}}\right] \mu_{p}(u)-c_{27}\|u\|^{r}[\operatorname{see}(80)] \\
& \geq c_{28}\|u\|^{p}-c_{27}\|u\|^{r} \text { for some } c_{28}>0 \\
& \left(\text { since } \eta<\widehat{\lambda}_{V} \text { and } \widehat{\lambda}_{V}>0\right) .
\end{aligned}
$$

Because $r>p$, choosing $\rho_{2} \in(0,1)$ small, we have

$$
\varphi_{p}(u)>0 \quad \text { for all } 0<\|u\| \leq \rho_{2}, u \in V .
$$

From (96) and (98), it follows that $\varphi_{p}$ has a local linking at the origin with respect to the decomposition (94). So, from Ref. 27, p. 171, we have $C_{1}\left(\varphi_{p}, 0\right) \neq 0$.

Now we are ready for the existence theorem for problem $(1)_{P}$.

Theorem 5. If hypotheses $H(\xi), H(\beta)$, and $H_{3}$ hold and $\widehat{\lambda}_{V}>0$, then problem $(1)_{P}$ admits a nontrivial solution $\widehat{u} \in C^{1}(\bar{\Omega})$.

Proof. From Proposition 11, we have that

$$
C_{1}\left(\varphi_{p}, 0\right) \neq 0 .
$$

Also, from Proposition 10, we know that

$$
C_{k}\left(\varphi_{p}, \infty\right)=0 \quad \text { for all } k \in \mathbb{N}_{0} .
$$

From (99) and (100), we infer that there exists $\widehat{u} \in W^{1, p}(\Omega)$ such that 


$$
\left.\widehat{u} \in K_{\varphi_{p}}, \widehat{u} \neq 0 \quad \text { (see Proposition } 4\right)
$$

$\Rightarrow \widehat{u}$ is a nontrivial solution of $(1)_{P}$.

The nonlinear regularity theory implies that $\widehat{u} \in C^{1}(\bar{\Omega})$.

We conclude with an existence result concerning the following nonlinear parametric Robin problem:

$$
(1)_{P}^{\lambda}\left\{\begin{array}{c}
-\Delta_{p} u(z)+\xi(z) u(z)^{p-1}=\lambda f_{0}(z, u(z)) \text { in } \Omega, \\
\frac{\partial u}{\partial n_{p}}+\beta(z) u^{p-1}=0 \text { on } \partial \Omega, u \geq 0 .
\end{array}\right.
$$

We impose the following conditions on the data of $(1)_{P}^{\lambda}$ :

$H_{0}: \xi \in L^{\infty}(\Omega), \beta \in C^{0, \alpha}(\partial(\Omega))$ with $\alpha \in(0,1), \xi \geq 0, \beta \geq 0$ and at least one of them is nontrivial.

$H_{4}: f_{0}: \Omega \times \mathbb{R} \rightarrow \mathbb{R}$ is a Carathéodory function such that $f_{0}(z, 0)=0$ for a.a. $z \in \Omega$, and hypotheses $H_{4}(\mathrm{i})$-(iii) are the same as the corresponding hypotheses $H_{1}(\mathrm{i})$-(iii) and

(iv) $\lim _{x \rightarrow 0^{+}} \frac{f_{0}(z, x)}{x^{p-1}}=0$ uniformly for a.a. $z \in \Omega$.

Remark 8. Hypothesis $H_{0}$ implies that $\widehat{\lambda}_{1}>0$. Then, hypothesis $H_{4}(i v)$ is a special case of hypothesis $H_{1}(i v)$.

Invoking Theorem 4, we have the following result concerning problem $(1)_{P}^{\lambda}$ :

Theorem 6. If hypotheses $H_{0}$ and $H_{4}$ hold, then for every $\lambda>0$ problem $(1)_{P}^{\lambda}$ admits a positive solution $\widehat{u}_{\lambda} \in D_{+}$.

Remark 9. For $p=2$ (semilinear problem) and Dirichlet boundary condition, with stronger hypotheses on the data, such an existence result is the main theorem in the work of Miyagaki-Souto. ${ }^{26}$ Their method of proof is more involved. Semilinear Robin problems were studied in the recent work of Hu-Papageorgiou. ${ }^{19}$

\section{REFERENCES}

${ }^{1}$ S. Aizicovici, N. S. Papageorgiou, and V. Staicu, "Degree theory for operators of monotone type and nonlinear elliptic equations with inequality constraints," Mem. Am. Math. Soc. 196, 70 (2008).

${ }^{2}$ S. Aizicovici, N. S. Papageorgiou, and V. Staicu, “Nodal solutions for ( $\left.p, 2\right)$-equations,” Trans. Am. Math. Soc. 367, 7343-7372 (2015).

${ }^{3}$ S. Barile and G. M. Figueiredo, "Existence of least energy positive, negative and nodal solutions for a class of $p \& q$-problems with potentials vanishing at infinity," J. Math. Anal. Appl. 427, 1205-1233 (2015).

${ }^{4}$ P. Candito, R. Livrea, and N. S. Papageorgiou, "Nonlinear elliptic equations with asymmetric asymptotic behavior at $\pm \infty$," Nonlinear Anal. Real World Appl. 32, 159-177 (2016).

${ }^{5}$ L. Cherfils and Y. Il'yasov, “On the stationary solutions of generalized reaction diffusion equations with p\&q-Laplacian," Commun. Pure Appl. Anal. 4, 9-22 (2005).

${ }^{6}$ S. Cingolani and M. Degiovanni, "Nontrivial solutions for $p$-Laplace equations with right-hand side having $p$-linear growth at infinity," Commun. Partial Differ. Equations 30, 1191-1203 (2005).

7J. I. Díaz and J. E. Saa, "Existence et unicité de solutions positives pour certaines équations elliptiques quasilinéaires," C. R. Acad. Sci. Paris Sér. I Math. 305, 521-524 (1987).

${ }^{8}$ J. Dugundji, Topology (Allyn \& Bacon, Boston, 1966).

${ }^{9}$ M. Filippakis, A. Kristály, and N. S. Papageorgiou, "Existence of five nonzero solutions with exact sign for a $p$-Laplacian equation," Discrete Contin. Dyn. Syst. 24, 405-440 (2009).

${ }^{10}$ G. Fragnelli, D. Mugnai, and N. S. Papageorgiou, "Positive and nodal solutions for parametric nonlinear Robin problems with indefinite potential," Discrete Contin. Dyn. Syst. 36, 6133-6166 (2016).

${ }^{11}$ T.-M. Gao and C.-L. Tang, “Existence of positive solutions for superlinear $p$-Laplacian equations,” Electron. J. Differ. Equations 2015 (40), 8.

${ }^{12}$ L. Gasiński and N. S. Papageorgiou, Nonlinear Analysis, Series on Mathematical Analysis and Applications Vol. 9 (Chapman and Hall/CRC Press, Boca Raton, 2006).

${ }^{13}$ L. Gasiński and N. S. Papageorgiou, "Existence and multiplicity of solutions for Neumann p-Laplacian-type equations,” Adv. Nonlinear Stud. 8, 843-870 (2008).

${ }^{14}$ L. Gasiński and N. S. Papageorgiou, “Dirichlet $(p, q)$-equations at resonance,” Discrete Contin. Dyn. Syst. 34, 2037-2060 (2014).

${ }^{15}$ L. Gasiński and N. S. Papageorgiou, "Nonlinear elliptic equations with a jumping reaction,” J. Math. Anal. Appl. 443, 1033-1070 (2016).

${ }^{16}$ L. Gasiński and N. S. Papageorgiou, Exercises in Analysis. Part 2: Nonlinear analysis, Problem Books in Mathematics (Springer, Cham, 2016).

${ }^{17}$ S. Hu and N. S. Papageorgiou, Handbook of Multivalued Analysis, Volume I: Theory, Mathematics and Its Applications Vol. 419 (Kluwer Academic Publishers, Dordrecht, The Netherlands, 1997).

${ }^{18}$ S. Hu and N. S. Papageorgiou, "Nonlinear Neumann equations driven by a nonhomogeneous differential operator," Commun. Pure Appl. Anal. 10, 1055-1078 (2011).

${ }^{19}$ S. Hu and N. S. Papageorgiou, "Positive solutions for Robin problems with general potential and logistic reaction," Commun. Pure Appl. Anal. 15, 2489-2507 (2016).

${ }^{20}$ L. Iturriaga, M. A. Souto, and P. Ubilla, “Quasilinear problems involving changing-sign nonlinearities without an Ambrosetti-Rabinowitz-type condition,” Proc. Edinb. Math. Soc. (2) 57, 755-762 (2014).

${ }^{21}$ L. Iturriaga, S. Lorca, and P. Ubilla, “A quasilinear problem without the Ambrosetti-Rabinowitz-type condition,” Proc. Roy. Soc. Edinburgh Sect. A 140, 391-398 (2010).

${ }^{22}$ G. M. Lieberman, “The natural generalization of the natural conditions of Ladyzhenskaya and Ural'tseva for elliptic equations," Commun. Partial Differ. Equations 16, 311-361 (1991). 
${ }^{23}$ S. Liu, "Existence of solutions to a superlinear p-Laplacian equation," Electron. J. Differ. Equations 2001(66), 6.

${ }^{24} \mathrm{G}$. Li and C. Yang, "The existence of a nontrivial solution to a nonlinear elliptic boundary value problem of $p$-Laplacian type without the Ambrosetti-Rabinowitz condition," Nonlinear Anal. 72, 4602-4613 (2010).

${ }^{25}$ S. A. Marano, S. J. N. Mosconi, and N. S. Papageorgiou, “Multiple solutions to ( $\left.p, q\right)$-Laplacian problems with resonant concave nonlinearity,” Adv. Nonlinear Stud. 16, 51-65 (2016).

${ }^{26}$ O. H. Miyagaki and M. A. S. Souto, "Superlinear problems without Ambrosetti and Rabinowitz growth condition," J. Differ. Equations 245, 3628-3638 (2008).

${ }^{27}$ D. Motreanu, V. Motreanu, and N. S. Papageorgiou, Topological and Variational Methods with Applications to Nonlinear Boundary Value Problems (Springer, New York, 2014).

${ }^{28}$ D. Mugnai and N. S. Papageorgiou, "Resonant nonlinear Neumann problems with indefinite weight," Ann. Sc. Norm. Super. Pisa Cl. Sci. (5) 11, 729-788 (2012).

${ }^{29}$ N. S. Papageorgiou and V. D. Rădulescu, "Qualitative phenomena for some classes of quasilinear elliptic equations with multiple resonance," Appl. Math. Optim. 69, 393-430 (2014).

${ }^{30}$ N. S. Papageorgiou and V. D. Rădulescu, "Multiple solutions with precise sign for nonlinear parametric Robin problems," J. Differ. Equations 256, 2449-2479 (2014).

${ }^{31}$ N. S. Papageorgiou and V. D. Rădulescu, "Bifurcation of positive solutions for nonlinear nonhomogeneous Robin and Neumann problems with competing nonlinearities," Discrete Contin. Dyn. Syst. 35, 5003-5036 (2015).

${ }^{32}$ N. S. Papageorgiou and V. D. Rădulescu, "Nonlinear nonhomogeneous Robin problems with superlinear reaction term," Adv. Nonlinear Stud. 16, 737-764 (2016).

${ }^{33}$ N. S. Papageorgiou, V. D. Rădulescu, and D. Repovs, "Positive solutions for perturbations of the Robin eigenvalue problem plus an indefinite and unbounded potential," Discrete Contin. Dyn. Syst. Ser. A 37, 2589-2618 (2017).

${ }^{34}$ N. S. Papageorgiou and V. D. Rădulescu, "Resonant (p, 2)-equations with asymmetric reaction," Anal. Appl. 13, 481-506 (2015).

${ }^{35}$ N. S. Papageorgiou and G. Smyrlis, "Positive solutions for parametric p-Laplacian equations," Commun. Pure Appl. Anal. 15, 1545-1570 (2016).

${ }^{36} \mathrm{P}$. Pucci and J. Serrin, The Maximum Principle (Birkhäuser Verlag, Basel, 2007).

${ }^{37}$ M. Sun, "Multiple solutions of a superlinear $p$-Laplacian equation without AR-condition," Appl. Anal. 89, 325-336 (2010).

${ }^{38} \mathrm{M}$. Sun, "Multiplicity of solutions for a class of the quasilinear elliptic equations at resonance," J. Math. Anal. Appl. 386, 661-668 (2012).

${ }^{39}$ M. Sun, M. Zhang, and J. Su, "Critical groups at zero and multiple solutions for a quasilinear elliptic equation," J. Math. Anal. Appl. 428, 696-712 (2015). 